Proc. Indian Acad. Sci. (Math. Sci.), Vol. 90, Number 3, July 1981, pp. 239-271.

(C) Printed in India.

\title{
Homogenization of eigenvalue problems in perforated domains
}

\author{
$M$ VANNINATHAN \\ TIFR Centre, Indian Institute of Science, Bangalore 560 012, India
}

MS received 9 December 1980 ; revised 3 January 1981

\begin{abstract}
In this paper, we treat some eigenvalue problems in periodically perforated domains and study the asymptotic behaviour of the eigenvalues and the eigenvectors when the number of holes in the domain increases to infinity. Using the method of asymptotic expansion, we give explicit formula for the homogenized coefficients and expansion for eigenvalues and eigenvectors. If we denote by $\epsilon$ the size of each hole in the domain, then we obtain the following aysmptotic expansion for the eigenvalues :

Dirichlet : $\quad \lambda_{\epsilon}=\epsilon^{-2} \lambda+\lambda_{0}+O(\epsilon)$,

Stekloff : $\quad \lambda_{\epsilon}=\epsilon \lambda_{1}+O\left(\epsilon^{2}\right)$,

Neumann : $\quad \lambda_{\epsilon}=\lambda_{0}+\epsilon \lambda_{1}+O\left(\epsilon^{2}\right)$.

Using the method of energy, we prove a theorem of convergence in each case considered here. We briefly study correctors in the case of Neumann eigenvalue problem.
\end{abstract}

Keywords. Homogenization ; correctors ; eigenvalues ; eigenvectors.

\section{Introduction}

The theory of homogenization has been developed by many authors in recent years. For a historic introduction and for a complete bibliography of the subject, the reader is referred to the book of Bensoussan et al [3]. The method of asymptotic development introduced in this book can also be applied to problems in a periodically perforated domain. For the treatment of homogenization problems in such domains, the reader is referred to the works of Lions [13], Duvaut [9], Gioranescu [7], Gioranescu and Saint Jean Paulin [8].

The study of such problems is important from theoretical as well as numerical point of view. Because of the complicated structure of the perforated domains, any kind of calculation is difficult to perform. For example, if we treat the Dirichlet problem, we have to impose the boundary condition on the boundary of the holes which are many in number. So, we wculd like to "approximate" the given problem by a "homogenized" problem on the domain without holes. 
By the method of asymptotic development, a problem on a periodically perforated domain is reduced to solving problems in the "basic cell" and in the domain without holes.

This paper is divided into three parts :

Part A : Dirichlet eigenvalue problem

Part B : Stekloff eigenvalue problem

Part C: Neumann eigenvalue problem.

Our aim is to describe the asymptotic behaviour of the various eigenvalues when the number of holes in the domain increases to infinity. In each case we explicitly write down the "homogenized operator" with the help of the method of asymptotic development and prove a homogenization theorem using the energy method introduced by Tartar [14] and prolongation operators of Cioranescu and Saint Jean Paulin [8].

We treat here the case of Laplacian operator. But one can extend the results to the case of elliptic, self-adjoint operators with periodic coefficients of the form

$$
A^{\epsilon} \equiv-\sum_{i=j} \frac{\partial}{\partial x_{i}}\left(a_{i j}(x / \epsilon) \frac{\partial}{\partial x_{j}}\right) .
$$

The eigenvalue problem corresponding to $A^{\epsilon}$ in a fixed domain has been studied by Kesavan [12]. The results of this paper were announced in Vanninathan [15], [16].

\section{Notations and hypothesis}

First, we consider a reference cell :

$$
Y=\prod_{i=1}^{N}\left(0, y_{i}^{0}\right) \subset \mathbf{R}^{N}, y_{i}^{0}>0 .
$$

Let $\tau_{i}(i=1,2, \ldots, M)$ be connected bounded open subsets of $\mathbf{R}^{N}$ with sufficiently smoath boundaries and which lie locally on one side of the boundary. Then the holes in $Y$ are $\bar{\tau}_{i} \cap Y(i=1,2, \ldots M)$ and their union is denoted by $T$ :

$$
T=\bigcup_{i=1}^{M}\left(\bar{\tau}_{i} \cap Y\right)
$$

Let

(2.3) $\quad Y^{*}=Y-T$.

Let $S$ denote the boundary of $T$ in $Y^{*}$. For details see figure 1 .

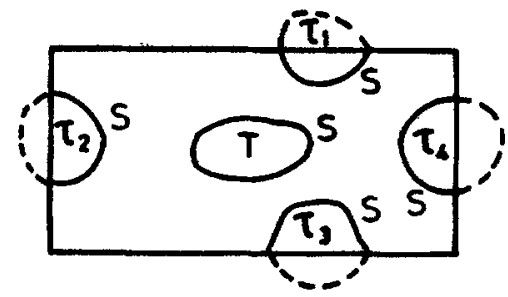

Figure 1 
We assume that

(H1) $\quad Y^{*}$ is connected.

For a function $f$ given on $Y$, we define the average of $f$ on $Y$ :

$$
m_{Y}(f)=\frac{1}{|Y|} \int_{Y} f(y) d y,
$$

where $|Y|$ denotes the Lebesgue measure of $Y$. Let $\epsilon$ be a small positive parameter which goes to zero. We denate by $f^{\epsilon}(x)$ or $f(x / \epsilon)$, the function defined in $\mathbf{R}^{N}$ in a periodic fashion with period $\epsilon y_{i}^{0}$ in the direction $x_{i}$.

Let us define now, "the holes" in $\mathbf{R}^{N}$ corresponding to $\epsilon$, starting from those in $Y$. For that we jntroduce the function $\chi$ as follows:

$$
\chi(y)=\left\{\begin{array}{lll}
1 & \text { if } & y \in Y^{*} \\
0 & \text { if } & y \in T .
\end{array}\right.
$$

We consider also the characteristic function of $T$ :

$$
\chi_{\Gamma}(y)=\left\{\begin{array}{lll}
1 & \text { if } & y \in T, \\
0 & \text { if } & y \in Y^{*}
\end{array}\right.
$$

Put

$$
\theta=m_{Y}(x)=\frac{\left|Y^{*}\right|}{|Y|}
$$

The "holes" in $\mathbf{R}^{N}$ are the connected components of the set

$$
\left\{x \in \mathbf{R}^{N} / \chi^{e}(x)=0\right\} \text {. }
$$

Finally, lat us be given a bounded connected open subset $\Omega$ of $\mathbf{R}^{N}$ whose boundary is sufficiently smooth and $\Omega$ lies locally on one side of its boundary. The "holes" in $\Omega$ are then defined by

(2.8) $T_{\theta}=$ connected components in $\Omega$ of $\left\{x \in \Omega / \chi^{\circ}(x)=0\right\}$. The perforated domain $\Omega_{e}$ with which we work is

$$
\Omega_{e}=\Omega-T_{e} \text {. }
$$

Let $S_{e}$ denote the boundary of $T_{e}$ in $\Omega_{\varepsilon}$.

We make the following restrictions on the geometry of $\Omega_{\mathrm{a}}$.

(H2) $\Omega_{e}$ is connected.

(H3) Each hole in $T_{e}$ has regular boundary.

In the problems we consider here, there is one more restriction on the geometry of $\Omega$ and the holes ( $c f$. Cioranescu and Saint Jean Paulin [8]).

(H4) The holes $T_{e}$ do not meet $\partial \Omega$, the boundary of $\Omega$.

We need, in fact, in Part A a stronger hypothesis. Given any hole $T^{\prime}$ in $Y$, we can as before construct the holes $T_{e}^{*}$ in $\Omega$ periodically. Set

(2.10) $\Omega_{T^{*}}=\Omega-T_{\mathrm{e}}^{\prime}$.

With this notation, it is evident that

(2.11) $\Omega_{\epsilon}=\Omega_{\tau_{\epsilon}}$.

The stronger hypothesis is the following :

(H5) $\left\{\begin{array}{l}\text { there exists a hole } T^{\prime} \text { in } Y \text { such that } T \subset \subset T^{\prime} \text { and the holes } T_{\varepsilon}^{\prime} \text { do } \\ \text { not intersect } \partial \Omega \text {. }\end{array}\right.$ 
Remark (2.1). The hypothesis ( $\mathrm{H} 4)$ is severe on the geometry of the domain $\Omega$ and the hole $T$. One such example of $\Omega$ is a finite union of cells homothetic to $Y$ and with the hole $T$ placed in the middle of $Y$.

Summation Convention. We adopt the usual summation convention with respect to the repeated indices.

\section{Part A; Dirichlet eigenvalue problem}

\section{Problem to be treated}

With the above notations, we consider the following eigenvalue problem:

$$
\left\{\begin{array}{l}
\text { Find }\left(u_{\epsilon}, \lambda_{e}\right) \in H_{0}^{1}\left(\Omega_{\epsilon}\right) \times \mathbf{R} \text { such that } \\
-\triangle u_{\epsilon}=\lambda_{\varepsilon} u_{e} \text { in } \Omega_{\epsilon} \\
\left(u_{\epsilon}, u_{\epsilon}\right)_{e}=1
\end{array}\right.
$$

where $(.,)_{e}$ denotes the inner product in $L^{2}\left(\Omega_{e}\right)$ and $H_{0}^{1}\left(\Omega_{e}\right)=\left\{v \in L^{2}\left(\Omega_{e}\right)\right.$; $\partial v / \partial x_{i} \in L^{2}\left(\Omega_{\epsilon}\right)$ for $i=1,2, \ldots N$ and $v=0$ on $\left.\partial \Omega\right\}$.

The variational formulation of this problem is the following :

$$
\left\{\begin{array}{l}
\text { Find }\left(u_{\epsilon}, \lambda_{\epsilon}\right) \in H_{0}^{1}\left(\Omega_{\epsilon}\right) \times \mathbf{R} \text { such that } \\
a^{\epsilon}\left(u_{\epsilon}, v\right)=\lambda_{\epsilon}\left(u_{\epsilon}, v\right)_{\epsilon} \text { for } v \in H_{0}^{1}\left(\Omega_{\epsilon}\right), \\
\left(u_{\epsilon}, u_{\epsilon}\right)_{\epsilon}=1,
\end{array}\right.
$$

with the bilinear form $a^{\epsilon}(.,$.$) defined by$

$$
a^{\varepsilon}(u, v)=\int_{\Omega_{e}} \frac{\partial u}{\partial x_{i}} \frac{\partial v}{\partial x_{i}} d x .
$$

According to spectral theory, there exist a sequence of eigenvalues $\left\{\lambda_{\epsilon}^{l}\right\}_{l=1}^{\infty}$ and a sequence of corresponding eigenvectors $\left\{u_{\varepsilon}^{l}\right\}_{l=1}^{\infty}$ such that

$$
\left\{\begin{array}{l}
0<\lambda_{\varepsilon}^{1}<\lambda_{\varepsilon}^{2} \leqslant \lambda_{e}^{3} \ldots \rightarrow \infty, \\
\lambda_{\varepsilon}^{l} \text { is of finite multiplicity for each } l, \\
\text { and }\left\{u_{\varepsilon}^{2}\right\}_{l=1}^{\infty} \text { form an orthonormal basis in } L^{2}\left(\Omega_{\varepsilon}\right) .
\end{array}\right.
$$

We can characterise the eigenvalues $\lambda_{e}^{2}$ with the help of Rayleigh quotient ( $c f$. Weinstein and Steinger [17]).

$$
R_{\epsilon}(v)=\frac{a^{e}(v, v)}{(v, v)_{\epsilon}} \text { for } v \in H_{0}^{1}\left(\Omega_{\epsilon}\right), v \neq 0
$$

The mimimax principle for the eigenvalues states that

$$
\left\{\begin{aligned}
\lambda_{e}^{l} & =\min \left\{\max _{v e S l} R_{\epsilon}(v) ; S_{l} \subset H_{0}^{i}\left(\Omega_{e}\right), \operatorname{dim} S_{l}=l\right\} \\
& =\max _{v e E_{e}(v)} R_{\epsilon}(v) \\
& =\max \left\{R_{e}(v) ;\left(v, u_{e}^{i}\right)_{e}=0, i=1,2, \ldots, l-1\right\}
\end{aligned}\right.
$$

where $E_{\epsilon}(l)$ is the subspace of $H_{0}^{1}\left(\Omega_{e}\right)$ spanned by $\left\{u_{e}^{1}, \ldots, u_{e}^{l}\right\}$.

This part is devoted to the study of the behaviour of $\lambda_{e}^{l}$ and $u_{e}^{l}$ when $\epsilon \rightarrow 0$. We prove, in particular, that $\lambda_{e}^{2}$ is of order $\epsilon^{-2}$ and that $\left\{\lambda_{e}^{2}-\epsilon^{-2} \lambda\right\} \rightarrow l$ th eigenvalue of the "homogenized problem" where $\lambda$ is the first eigenvalue in the cell 
$Y^{*}$. As we will see, some weighted Sobolev spaces and their propertits are used in this study.

\section{Eigenvalue problem in the cell $Y^{*}$}

Let us define the space

$$
\begin{aligned}
& W_{0}=\left\{v \in H^{1}\left(Y^{*}\right) ; v=0 \text { on } S \text { and } v \text { is } Y \text {-periodic, i.e., } v\right. \text { assumes } \\
& \text { same values on the opposite faces of } Y\}
\end{aligned}
$$

and the bilinear form

$$
a(u, v)=\int_{Y_{*}} \frac{\partial u}{\partial y_{i}} \frac{\partial v}{\partial y_{i}} d y \text { for } u, v \in H^{1}\left(Y^{*}\right) .
$$

The bilinear form being elliptic on $W_{0}$, the following problem is well posed :

$$
\left\{\begin{array}{l}
\text { Find }(\phi, \lambda) \in W_{0} \times \mathbf{R} \text { such that } \\
a(\phi, v)=\lambda(\phi, v)_{Y *} \text { for } v \in W_{0}, \\
(\phi, \phi)_{Y *}=1,
\end{array}\right.
$$

where $(., .)_{Y *}$ denotes the scalar product in $L^{2}\left(Y^{*}\right)$.

In what follows, we consider only the first eigenvalue $\lambda$ of the above problem. It is well known that $\lambda$ is simple and the corresponding eigenvector $\phi$ has constant sign in $Y^{*}$. We choose the vector $\phi$ which is uniquely defined by (4.3) and

$$
\phi>0 \text { in } Y^{*} \text {. }
$$

Remark (4.1). We extend $\phi$ by zero in the interior of the holes $T$ and we denote again by $\phi$ the extended function.

Remark (4.2). It follows from (4.3) that the function $\phi^{\epsilon}$ defined periodically satisfies

$$
\left\{\begin{array}{l}
-\triangle \phi^{\epsilon}=\epsilon^{-2} \lambda \phi^{\epsilon} \text { in } \Omega_{e}, \\
\phi^{\epsilon}=0 \text { on } S_{\epsilon} .
\end{array}\right.
$$

However, $\phi^{\mathbf{e}}$ is not zero on $\partial \Omega$.

\section{Some weighted Sobolev spaces}

We will see later that consideration of some weighted Sobolev spaces is very important in the study of the present problem. There is a vast literature on this subject: see for example Baouendi [1], Baouendi and Goulaouic [2], Geymont and Grisvard [10], Goudjo [11]. In this section, we define so me weighted Sobolev spacts and state some of their properties which will be needed later.

We consider the following spaces with weights $\phi^{\epsilon}$ and $\phi$ ( $\phi$ being defined in $\left.\S 4\right)$ :

$$
\begin{aligned}
V_{\epsilon} & =\left\{v \in D^{\prime}\left(\Omega_{e}\right) ; \phi^{\epsilon} v \in L^{2}\left(\Omega_{\epsilon}\right), \phi^{\epsilon} \frac{\partial v}{\partial x_{i}} \in L^{2}\left(\Omega_{e}\right)\right. \text { for } \\
i & =1,2, \ldots, N \text { and } v=0 \text { on } \partial \Omega\}
\end{aligned}
$$




$$
\begin{aligned}
& V=\left\{v \in D^{\prime}\left(Y^{*}\right) ; \phi v \in L^{2}\left(Y^{*}\right), \phi \frac{\partial v}{\partial y_{4}} \in L^{2}\left(Y^{*}\right)\right. \text { for } \\
& j=1,2, \ldots, N \text { and } v \text { is } Y \text {-periodic }\} .
\end{aligned}
$$

where, as usual,

$D^{\prime}(\Omega)=$ space of distributions on $\Omega$, and these spaces are provided with the following norms :

$$
\begin{aligned}
& \|v\|_{\varepsilon}=\left[\left\|\phi^{\epsilon} v\right\|_{L^{2}\left(\Omega_{e}\right)}^{\varepsilon^{2}}+\sum_{i=1}^{N}\left\|\phi^{\epsilon} \frac{\partial v}{\partial x_{i}}\right\|_{L^{2}\left(\Omega_{e}\right)}^{2}\right]^{1 / 2}, \\
& \|v\|_{v}=\left[\|\phi v\|_{L^{2}\left(Y^{*}\right)}^{2}+\sum_{i=1}^{N}\left\|\phi \frac{\partial v}{\partial y_{l}}\right\|_{L^{2}\left(Y^{*}\right)}^{2}\right]^{1 / 2} .
\end{aligned}
$$

Some of their propertieis are given in the following :

\section{Proposition (5.1)}

(1) The space $D\left(\Omega_{e}\right)$ of infinitely differentiable functions on $\Omega_{\epsilon}$ with compact support in $\Omega_{\varepsilon}$ is dense in $V_{\mathrm{e}}$.

(2) We have a continuous inclusion

$$
V_{e} \rightarrow L^{2}\left(\Omega_{e}\right)
$$

and the continuity constant of this inclusion does not depend on $\epsilon$ : there exists a constant $c>0$ independent of $\epsilon$ such that

$$
\|v\|_{L^{2}\left(\Omega_{e}\right)} \leq c\|v\|_{e} \forall v \in V_{e} .
$$

(3) The map $v \rightarrow \phi^{\epsilon} v$ defines an isomorphis m of $V_{e}$ onto $H_{o}^{1}\left(\Omega_{\epsilon}\right)$.

(4) The inclusion $V_{e} \rightarrow L^{2}\left(\phi^{\epsilon}\right)$ is compact where

$$
L^{2}\left(\phi^{\epsilon}\right)=\left\{v \in D^{\prime}\left(\Omega_{\varepsilon}\right) ; \phi^{\epsilon} v \in L^{2}\left(\Omega_{e}\right)\right\}
$$

$$
v \rightarrow\left[\sum_{i=1}^{N}\left\|\phi^{e} \frac{\partial v}{\partial x_{i}}\right\|_{L^{2}\left(\Omega_{e}\right)}^{2}\right]^{1 / 2}
$$

defines a norm on $V_{e}$ equivalent to the norm \|\|$_{e}$.

Proof. All these properties are proved in the works cited above. The only thing which is now is the inequality (5.5) with $c$ independent of $\epsilon$. But one can prove this, without much difficulty, from the continuity of the inclusion

$$
W(\phi) \rightarrow L^{2}\left(Y^{*}\right)
$$

where the space $W(\phi)$ is defined by

$$
W(\phi)=\left\{v \in D^{\prime}\left(Y^{*}\right) ; \phi v \in L^{2}\left(Y^{*}\right), \phi \frac{\partial v}{\partial y_{i}} \in L^{2}\left(Y^{*}\right) \text { for } i=1,2, \ldots, N .\right\}
$$




\section{Proposition (5.2)}

(1) The space of functions $v \in c^{\infty}\left(\bar{Y}^{*}\right) \cap V$ which vanish in a neighbourhood of $S$ are dense in $V$.

(2) One has the continuous inclusion

$$
V \rightarrow L^{2}\left(Y^{*}\right) \text {. }
$$

(3) The map $v \rightarrow \phi v$ defines an isomorphism of $V$ onto $W_{0}$.

(4) The inclusion

(5.9) $\quad V \rightarrow L^{2}(\phi)$

is compact where we define

$$
L^{2}(\phi)=\left\{v \in D^{\prime}\left(Y^{*}\right) ; \phi v \in L^{2}\left(Y^{*}\right)\right\}
$$

$$
v \rightarrow\left[\sum_{i=1}^{N}\left\|\phi \frac{\partial v}{\partial y_{i}}\right\|_{L^{2}\left(x^{*}\right)}^{2}\right]^{1 / 2}
$$

defines a norm equivalent to the quotient norm on $V / \mathbf{R}$.

Now, we formulate the eigenvalue problems in the spaces $V_{e}$. We define

$$
\left\{\begin{array}{l}
a\left(\phi^{\epsilon} ; u, v\right)=\int_{\Omega_{e}} \phi^{\epsilon^{2}} \frac{\partial u}{\partial x_{i}} \frac{\partial v}{\partial x_{i}} d x \text { for } u, v \in V_{\epsilon}, \\
\left(\phi^{\epsilon} ; u, v\right)=\int_{\Omega_{e}} \phi^{\epsilon^{2}} u v d x \forall u, v \in L^{2}\left(\phi^{\epsilon}\right) .
\end{array}\right.
$$

By virtue of the properties (4) and (5) of the proposition (5.1), the following problem is well posed:

$$
\left\{\begin{array}{l}
\text { Find }\left(v_{e}, \mu_{e}\right) \in V_{e} \times \mathbf{R} \text { such that } \\
a\left(\phi^{\epsilon} ; v_{e}, v\right)=\mu_{e}\left(\phi^{\epsilon} ; v_{e}, v\right) \text { for } v \in V_{\epsilon} \\
\left(\phi^{\epsilon} ; v_{e}, v_{e}\right)=1
\end{array}\right.
$$

Let $\left\{u_{e}^{l}\right\}_{l=1}^{\infty}$ be the sequence of eigenvalues and $\left\{v_{e}^{l}\right\}_{l=1}^{\infty}$ the sequence of corresponding vectors satisfying

$$
\left\{\begin{array}{l}
\text { (i) } 0<\mu_{e}^{1} \leq \mu_{e}^{2} \leq \ldots \rightarrow \infty, \\
\text { (ii) }\left\{v_{e}^{l}\right\}_{l=1}^{\infty} \text { form an orthonormal basis in } L^{2}\left(\phi^{\epsilon}\right) .
\end{array}\right.
$$

We have once again the characterization of $\mu_{\mathrm{E}}^{l}$, analogous to (3.6), in terms of Rayleigh quotient

$$
R\left(\phi^{\epsilon} ; v\right)=\frac{a\left(\phi^{\epsilon} ; v, v\right)}{\left(\phi^{\epsilon} ; v, v\right)} \text { for } v \in V_{\epsilon}, v \neq 0
$$

\section{Estimations on the eigenvalues}

The following Lemma establishes one important relation between the eigenvalues $\lambda_{e}, \lambda$ and $\mu_{e}$. 
Lemma (6.1). Let $\lambda$ be the first eigenvalue of the problem (4.3) with the eigenvictor $\phi$. Then for all $v \in V_{\epsilon}$, we have

$$
\int_{\Omega_{e}} \frac{\partial}{\partial x_{i}}\left(\phi^{\epsilon} v\right) \frac{\partial}{\partial x_{i}}\left(\phi^{\epsilon} v\right) d x=\epsilon^{-2} \lambda \int_{\Omega_{e}} \phi^{\epsilon^{2}} v^{2} d x+\int_{\Omega_{e}} \phi^{\epsilon^{2}} \frac{\partial v}{\partial x_{i}} \frac{\partial v}{\partial x_{i}} d x
$$

Proof. Since $D\left(\Omega_{e}\right)$ is dense in $V_{e}$, it suffices to verify (6.1) for all $v \in D\left(\Omega_{e}\right)$. On the one hand, we have

$$
\int_{\Omega_{e}} \frac{\partial}{\partial x_{i}}\left(\phi^{\epsilon} v\right) \frac{\partial}{\partial x_{i}}\left(\phi^{\epsilon} v\right)=\int_{\Omega_{\epsilon}} \frac{\partial \phi^{\epsilon}}{\partial x_{i}} \frac{\partial}{\partial x_{i}}\left(\phi^{\epsilon} v^{2}\right) d x+\int_{\Omega_{\epsilon}} \phi \epsilon^{2} \frac{\partial v}{\partial x_{i}} \frac{\partial v}{\partial x_{i}} d x
$$

and on the other hand, by multiplying (4.5) by $\phi^{\epsilon} v^{2}$ we obtain

$$
\int_{\Omega_{e}} \frac{\partial \phi^{\epsilon}}{\partial x_{i}} \frac{\partial}{\partial x_{i}}\left(\phi^{\epsilon} v^{2}\right) d x=\epsilon^{-2} \lambda \int_{\Omega_{e}} \phi^{\epsilon^{2}} v^{2} d x
$$

and so we deduce (6.1) without difficulty.

Corollary (6.1)

Let $\left\{\lambda_{\varepsilon}^{l}\right\}_{l=1}^{\infty},\left\{\mu_{e}^{l}\right\}_{l=1}^{\infty}$ be the sequences of eigenvalues of the problems (3.2) and (5.12) respectively. Let $\lambda$ be the first eigenvalue of the problem (4.3). Then we have

$$
\lambda_{e}^{2}=\epsilon^{-2} \lambda+\mu_{e}^{2} \text { for } l \geq 1 \text {. }
$$

Proof. In fact, we obtain, from (6.1)

$$
R_{e}\left(\phi^{\epsilon} v\right)=\epsilon^{-2} \lambda+R\left(\phi^{\epsilon} ; v\right) \text { for } v \in V_{\epsilon} .
$$

Now we use the minimax principles for eigenvalues and the isomorphism of the proposition (5.1) (iii) to get the relation (6.2).

Since $\mu_{\epsilon}^{l}>0$ for all $l \geq 1$ and $\epsilon>0$, we see that the sequence $\left\{\lambda_{\varepsilon}^{l}-\epsilon^{-2} \lambda\right\}_{\epsilon>0}$ is bounded below by zero. The following Proposition shows that it is bounded above.

Proposition (6.1). Let $\left\{\mu_{e}^{l}\right\}_{l=1}^{\infty}$ be the sequence of eigenvalues of the problem (5.12). Then for $l \geq 1,\left\{\mu_{e}^{l}\right\}_{e>0}$ is bounded independently of $\epsilon$.

Proof. We use the following characterizations of minimax principle :

$$
\mu_{e}^{l}=\min \left\{\max _{v \in S l} R\left(\phi^{\epsilon} ; v\right) ; S_{\imath} \subset V_{\epsilon}, \operatorname{dim} S_{\imath}=l\right\}
$$

We take $S_{l}$ to be the vector space spanned by $w_{1}, w_{2}, \ldots, w_{l}$, the first $l$-eigenve:tors of the following Dirichlet problem :

$$
\left\{\begin{array}{l}
\text { Find }(w, v) \in H_{0}^{1}(\Omega) \times \mathbf{R} \text { such that } \\
-\triangle w=v w \text { in } \Omega .
\end{array}\right.
$$

It is not difficult to see that we have

$$
\operatorname{dim}\left(S_{l} / \Omega_{e}\right)=l \text { for } \epsilon>0 .
$$


$\left[S_{l} / \Omega_{\epsilon}\right.$ denotes the restriction to $\Omega_{\epsilon}$ of functions belonging to $\left.S_{l}\right]$. So, one can take $S_{l} / \Omega_{\epsilon}$ in (6.4). (This is alright, since no boundary condition is required on $S_{\epsilon}$ for $\left.V_{\epsilon}.\right)$ We obtain

(6.7) $\quad \mu_{\varepsilon}^{l} \leq \max _{\text {veS } l} R\left(\phi^{\epsilon} ; v\right)$.

We claim that the right hand side of the inequality (6.7) is bounded above by a constant independent of $\epsilon$. In fact, on the contrary we would have, for a sequence $\epsilon_{n} \rightarrow 0$, a sequeace $\left\{v_{n}\right\} \subset S_{z}$ such that

$$
\int_{\Omega_{n}} \phi_{n}^{2} \nabla v_{n} \nabla v_{n}>n \int_{\Omega_{n}} \phi_{n}^{2} v_{n}^{2} \text { for } n \geq 1,
$$

(6.9) $\quad \int_{\Omega} v_{s}^{2}=1$ fir $n \geq 1$.

Here we have set $\Omega_{e_{n}}=\Omega_{n}$ and $\phi^{\epsilon_{n}}=\phi_{n}$. Since $S_{l}$ is of finite dimension, we have (for a subsequence)

$$
v_{n} \rightarrow v \text { in } H_{0}^{1}(\Omega) \text { strong. }
$$

Now, one can pass to the limit in (6.8) and obtain

$$
m_{v}\left(\phi^{2}\right) \int_{\Omega} v^{2}=0 .
$$

But (6.9) implies that

$$
\int_{\Omega} v^{2}=1 \text {. }
$$

This contradiction proves the Proposition.

\section{Asymptotic development}

The aim of this section is to find "the homogenized operator" for the problem (3.2) by the method of asymptotic development introduced in Bensoussan et al [3] and Lions [13].

We introduce one "fast" variable :

(7.1) $y=x / \epsilon$.

Then, the differential operator $\partial / \partial x_{j}$ applied to a function $\phi(x, y)$ becomes

(7.2) $\frac{\partial}{\partial x_{j}}+\epsilon^{-1} \frac{\partial}{\partial y_{j}}$.

So, the laplacian operator is transformed into

$$
\epsilon^{-2} \triangle_{y}+2 \epsilon^{-1} \triangle_{a y}+\triangle_{.}
$$

where

$$
\Delta_{y}=\frac{\partial^{2}}{\partial y_{i} \partial y_{i}}, \quad \Delta_{e y}=\frac{\partial^{2}}{\partial x_{i} \partial y_{i}}, \quad \Delta_{l}=\frac{\partial^{2}}{\partial x_{i} \partial x_{i}} .
$$


Taking into account Proposition (6.1) and Corollary (6.1), we propose the following Ansatz for the problem (3.1) :

$$
u_{\epsilon}(x)=u_{0}(x, y)+\epsilon u_{1}(x, y)+\ldots y=x / \epsilon,
$$

$$
\lambda_{e}=\epsilon^{-2} \lambda_{-2}+\epsilon^{-1} \lambda_{-1}+\lambda_{0}+\ldots \text {. }
$$

We impose the following restrictions on the functions $u_{1}$ which are defined for $x \in \Omega$ and $y \in Y^{*}$ :

$$
\left\{\begin{array}{l}
u_{1}(x, y)=0 \text { if } y \in S, \\
u_{j}(x, .) \text { is Y-periodic in } y .
\end{array}\right.
$$

We substitute the expressions (7.5) and (7.6) in equation (3.1) and we identify the powers of $\epsilon$. We obtain

$$
\left\{\begin{array}{l}
u_{0}(x, y)=\phi(y) \tilde{u}_{0}(x), \\
u_{1}(x, y)=\phi^{(f)}(y) \frac{\partial \tilde{u}_{0}}{\partial x_{j}}(x)+\phi(y) \tilde{u}_{1}(x),
\end{array}\right.
$$

(7.9) $\lambda_{-2}=\lambda$,

(7.10) $\lambda_{-1}=0$,

(7.11) $A \tilde{u}_{0}=\lambda_{0} \tilde{u}_{0}$,

where

(1) $\lambda$ is the first eigenvalue and $\phi$ the corresponding eigenvector of the problem (4.3).

(2) The functions $\phi^{(j)}(j=1,2, \ldots, N)$ are defined by

(7.12) $\quad a\left(\phi^{(3)}, v\right)-\lambda\left(\phi^{(1)}, v\right)_{Y^{*}}=2\left(\frac{\partial \phi}{\partial y_{1}}, v\right)_{Y^{*}}$ for $v \in W_{0}, \phi^{(3)} \in W_{0}$.

(3) The operator $A$ (called homogenized operator) is defined by

(7.13) $A \equiv-q_{u} \frac{\partial^{2}}{\partial x_{i} \partial x_{j}}$

the "homogenized coefficients" being defined by

(7.14) $q_{i j}=\delta_{i j}+2 \int_{\gamma^{*}} \frac{\partial \phi^{(3)}}{\partial y_{i}} \phi d y$ for $i, j=1,2, \ldots, N$.

\section{Remark (7.1)}

The relations (7.9) and (7.10) are in accordance with the results of Proposition (6.1) and Corollary (6.1).

Remark (7.2) Since

$$
\left(\frac{\partial \phi}{\partial y_{i}}, \phi\right)_{Y^{*}}=0 \text { for } i=1,2, \ldots, N
$$

equations (7.12) can be solved for $\phi^{(j)}$ by Fredholm alternative. 
The "homogenized problem" is an eigenvalue problem for the operator $A$ :

$$
\left\{\begin{array}{l}
\text { Find }(u, \mu) \in H_{0}^{\perp}(\Omega) \times \mathbf{R} \text { such that } \\
A u=\mu u \text { in } \Omega \\
u \neq 0 .
\end{array}\right.
$$

The preceding formal calculations show that the sequence $\left\{\lambda_{\varepsilon}-\epsilon^{-2} \lambda\right\}_{\epsilon}>0$ converges to an eigenvalue of the problem (7.15). We prove this result later by the method of energy.

\section{Ellipticity of the homogenized operatar}

The idea of proving the ellipticity of the operator consists of identifying the coeffcients $q_{i j}$ with the homogenized coefficients associated with the problem (5.12). So, we apply the asymptotic development method to the problem (5.12). First, we write the problem (5.12) in operator form : The solution $\left(v_{\epsilon}, \mu_{\epsilon}\right)$ is characterized by

$$
\left\{\begin{array}{l}
\left(v_{e}, \mu_{\varepsilon}\right) \in V_{\varepsilon} \times \mathbf{R}, \\
-\frac{\partial}{\partial x_{i}}\left(\phi^{\varepsilon^{2}} \frac{\partial v_{\varepsilon}}{\partial x_{i}}\right)=\mu_{e} \phi^{\epsilon^{2}} v_{\varepsilon} \text { in } \Omega_{\varepsilon}, \\
\int_{\Omega_{e}} \phi^{\epsilon^{2}} v_{\varepsilon}^{2}=1 .
\end{array}\right.
$$

We develop $v_{\epsilon}$ and $\mu_{\epsilon}$ in the following form :

$$
\begin{aligned}
& v_{\epsilon}(x)=v_{0}(x, y)+\epsilon v_{1}(x, y)+\ldots y=x / \epsilon, \\
& \mu_{\epsilon}=\mu_{0}+\epsilon \mu_{1}+\ldots,
\end{aligned}
$$

where $v$, is defined on $\Omega \times Y^{*}$ and it is $Y$-periodic in $y$.

We put these expressions in (8.1) and identify the powers of $\epsilon$. We get the following results :

$$
\begin{aligned}
& v_{0}(x, y)=\tilde{v}_{0}(x) \\
& v_{1}(x, y)=\psi^{(j)}(y) \frac{\partial \tilde{v}_{0}}{\partial x_{1}}(x)+\tilde{v}_{1}(x)
\end{aligned}
$$

and the necessary and sufficient condition so that we solve for $v_{2}$ is

$$
B \bar{v}_{0}=\mu_{0} \tilde{v}_{0} \text { in } \Omega,
$$

where the operator $B$ is defined by

$$
B \equiv-p_{i j} \frac{\partial^{2}}{\partial x_{i} \partial x_{j}}
$$

with

$$
p_{i j}=\delta_{i j}+\int_{\gamma^{*}} \phi^{2} \frac{\partial \psi^{(j)}}{\partial y_{i}} d y \text { for } i, j=1,2, \ldots, N
$$


The functions $\psi^{(i)}(j=1,2, \ldots, N)$ are defined (upto an additive constant) to be solutions of the following variational problem :

$$
\left\{\begin{array}{l}
a\left(\phi ; \psi^{(j)}, v\right)=-a\left(\phi ; y_{j}, v\right) \forall v \in V, \\
\psi^{(j)} \in V,
\end{array}\right.
$$

the bilinear form $a(\phi ;, .$.$) being defined by$

$$
a(\phi ; u, v)=\int_{Y^{*}} \phi^{2} \frac{\partial u}{\partial y_{i}} \frac{\partial v}{\partial y_{i}} d y \forall u, v \in V
$$

It is not difficult to see that the operator $B$ is elliptic in the sense that there exists a constant $a>0$ such that

$$
p_{i j} \xi_{i} \xi_{j} \geq a \xi_{i} \xi_{i} \text { for } \xi=\left(\xi_{i}\right) \in \mathbf{R}^{N} \text {. }
$$

In fact, the coefficients $p_{i j}$ can be expressed by the following formula :

$$
p_{i j}=a\left(\phi ; \psi^{(j)}+y_{i}, \psi^{(i)}+y_{i}\right) \text { for } i, j=1,2, \ldots N,
$$

taking into account the following relation

$$
a\left(\phi ; \psi^{(j)}+y_{i}, \psi^{(i)}\right)=0 \text { for } i, j=1,2, \ldots N \text {. }
$$

Now, the inequality (8.11) is a simple consequence of (8.12).

Theorem (8.1)

Let $\left(q_{i j}\right)$ and $\left(p_{i j}\right)$ be defined by (7.14) and (8.8) respectively. Then we have

$$
q_{i j}=p_{i j} \text { for } i, j=1,2, \ldots N .
$$

In particular, the operator $A$ is symmetric and elliptic.

Proof. We prove that $\phi \psi^{(j)}$ is a sclution to the problem (7.12). For that we use the isomorphism between the spaces $V$ and $W_{0}$ given by the Proposition (5.2) (iii). Firstly, we have $\phi \psi^{(j)} \in W_{0}$.

By vit tue of the Proposition (5.2) (i), it suffices to verify that

$$
\int_{Y^{*}} \frac{\partial}{\partial y_{i}}\left(\phi \psi^{(j)}\right) \frac{\partial}{\partial y_{i}}(\phi v) d y=\lambda \int_{Y^{*}} \phi^{2} \psi^{(j)} v d y+2 \int_{Y^{*}} \frac{\partial \phi}{\partial y_{j}} \phi v d y,
$$

for all $v \in c^{\infty}\left(\bar{Y}^{*}\right) \cap V$ which vanish in a neighbourhood of $S$. The relation (8.15) is easily proved by using the definitions of $\phi$ and $\psi^{(j)}$ and the following identity :

$$
2 \int_{Y^{*}} \phi \frac{\partial \phi}{\partial y_{i}} v d y=-\int_{Y^{*}} \phi^{2} \frac{\partial v}{\partial y_{i}} d y \forall v \in V
$$

Now we take $\phi^{(j)}=\phi \psi^{(j)}$ in the formula $(7.14)$ defining $q_{i j}$. We obtain

$$
q_{i j}=\delta_{i j}+2 \int_{Y^{*}} \frac{\partial}{\partial y_{i}}\left(\phi \psi^{(j)}\right) \phi d y
$$




$$
\begin{aligned}
& =\delta_{i j}+\int_{Y^{*}} \phi^{2} \frac{\partial \psi^{(s)}}{\partial y_{i}} d y \\
& =p_{i j},
\end{aligned}
$$

and so the Theorem is proved.

\section{Homogenization thearems}

In this section using energy method we show how one passes to the limit as $\epsilon \rightarrow 0$ in the problem (5.12) which determines the correctors $\mu_{\epsilon}$. Before that, we need some more notation;. Let us denote by $T^{\prime}$ any hole satisfying the hypothesis (H5). We denote by $P_{e}^{T^{\prime}}$, the prolongation operator constructed from the hole $T^{\prime}$ satisfying the following condition :

$$
\left\{\begin{array}{l}
\text { For all } v \text { in } H^{1}\left(\Omega_{T_{\epsilon}^{\prime}}\right) \text { with } v=0 \text { on } \partial \Omega \text {, we have } \\
P_{\epsilon}^{T^{\prime}} v \in H_{0}^{1}(\Omega) \text { and there exists a constant } c>0 \\
\text { independent of } \left.\epsilon \text { (but depending on the hole } T^{\prime}\right) \text { such that } \\
\left|P_{\varepsilon}^{T^{\prime}} v\right|_{1, \Omega} \leq c|v|_{1, \Omega_{T_{\epsilon}^{\prime}}} \\
\text { where }|v|_{1, \Omega}=\int_{\Omega} \nabla v \cdot \nabla v d x .
\end{array}\right.
$$

The existence of the operator $P_{E}^{T^{\prime}}$ is proved in Cioranescu and Saint Jean Paulin [8] and Cioranescu [7].

Lemma (9.1). Suppose for each $\epsilon>0$, we are given $w_{\epsilon}$ in $V_{\epsilon}$ such that (9.2) $\left\|w_{\epsilon}\right\|_{\epsilon} \leqslant c$, independent of $\epsilon$.

Then there exists a subsequence of $\epsilon$ (again denoted by $\epsilon$ ) and a function $w_{0}$ in $H_{0}^{1}(\Omega)$ such that

(9.3) $P_{\varepsilon}^{T^{\prime}} w_{\epsilon} \rightarrow w_{0}$ in $H_{0}^{1}(\Omega)$ weak,

for all holes $T^{\prime}$ verifying (H5).

Proof. It follows from the hypothesis (9.2) and from the fact that $\phi>0$ in $Y^{*}$ that there exists a constant $c_{T^{\prime}}$ such that

(9.4) $\left|w_{\epsilon}\right|_{1, \Omega_{T_{\epsilon}^{\prime}}} \leq c_{T^{\prime}}$.

Thanks to the inequality (9.1), we see that the sequence $\left\{P_{e}^{T^{\prime}} w_{\epsilon}\right\}$ remains bounded in $H_{0}^{1}(\Omega)$. So, wa can extract a subsequence of $\epsilon$ and a function $w_{0}^{T^{\prime}}$ in $H_{0}^{1}(\Omega)$ both depending on $T^{\prime}$ such that

$$
P_{e}^{T \prime} w_{\epsilon} \rightarrow w_{0}^{T^{*}} \text { in } H_{0}^{1}(\Omega) \text { weak, }
$$

for the extracted subsequence.

Now lat ur consider another hole sarisfying (H5). By similar arguments as before, we obtain a subsequence of the substquence already picked up and a function $w_{0}^{T^{\prime \prime \prime}}$ in $H_{0}^{1}(\Omega)$ such that 
(9.6) $\quad P_{\varepsilon}^{T^{\prime \prime}} w_{\epsilon} \rightarrow w_{0}^{T \prime \prime}$ in $H_{0}^{1}(\Omega)$ weak.

But we have the relation

$$
\chi_{Y-\left(T^{\prime} \cup T^{\prime \prime}\right)}(x / \epsilon) P_{e}^{T^{\prime \prime}} w_{\epsilon}=\chi_{Y-\left(T^{\prime} \cup T^{\prime \prime}\right)}(x / \epsilon) P_{e}^{T^{\prime \prime \prime}} w_{\epsilon} \text { in } \Omega
$$

where $\chi_{Y-\left(T^{\prime} \cup T^{\prime \prime}\right)}(y)$ is the characteristic function of $Y-\left(T^{\prime} \cup T^{\prime \prime}\right)$. One can pass to the limit in (9.7) and we obtain

$$
m_{Y}\left(\chi_{Y-\left(T^{\prime} \cup T^{\prime \prime \prime}\right)}\right) w_{0}^{T^{\prime}}=m_{Y}\left(\chi_{Y-\left(T^{\prime} \cup T^{\prime \prime}\right)}\right) w_{0}^{T^{\prime \prime \prime}},
$$

and so $w_{0}^{T \prime}=w_{0}^{T^{\prime \prime}}$ in $\Omega$. By the uniqueness of the limit, we see that the proof is complete.

Lemma (9.2). Let $\left\{w_{\epsilon}\right\}_{\epsilon}>_{0}$ be given as in the preceding Lemma. Then for the subsequence of $\epsilon$ and for the function $w_{0}$ in $H_{0}^{1}(\Omega)$ given by the Lemma $(9.1)$ we have

(9.8) $\quad P_{e}^{T^{\prime}} w_{\epsilon} \rightarrow w_{0}$ in $H_{0}^{1}(\Omega)$ weak for all holes $T^{\prime}$,

(9.9) $\int_{\Omega_{\epsilon}} \phi(x / \epsilon) f(x / \epsilon) w_{\epsilon} d x \rightarrow \int_{a} m_{Y}(\phi f) w_{0} d x$,

where $f$ is given in $L^{2}(Y)$.

Proof. Let $T^{\prime}$ be a hole satisfying (H5). We write

(9.10) $\int_{\Omega e} \phi^{\epsilon} f^{\epsilon} w_{\epsilon} d x-\int_{\Omega} m_{Y}(\phi f) w_{0} d x$

$$
\begin{aligned}
=[ & \left.\int_{\Omega_{T_{\epsilon}^{\prime}}} \phi^{\epsilon} f^{\epsilon} w_{\epsilon} d x-\int_{\Omega} m_{Y-T^{\prime}}(\phi f) w_{0}\right]+\left[\int_{\Omega_{\epsilon}-\Omega_{T_{\epsilon}^{\prime}}} \phi^{\epsilon} f^{\epsilon} w_{\epsilon} d x\right. \\
& \left.-\int_{\Omega} m_{T^{\prime}-\tau}(\phi f) w_{0} d x\right],
\end{aligned}
$$

where we have set

(9.11) $m_{U}(g)=\frac{1}{|Y|} \int_{U} g d y$ for subsets $U$ of $Y$.

We have the following estimate :

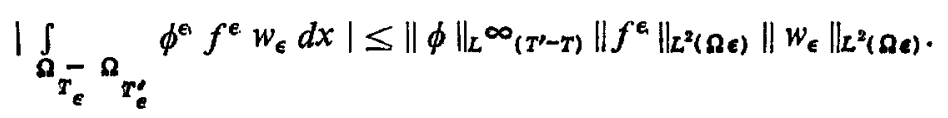

Using now Proposition (5.1) (2), we deduce

(9.12) $\left|\int_{\Omega_{T_{e}}-\Omega_{r_{\varepsilon}^{*}}} \phi^{e} f^{\epsilon} w_{\epsilon} d x\right| \leq c\|\phi\|_{L}^{\infty}\left(r^{\prime}-r\right)$,

where $c$ is a constant independent of $T^{\prime}$ and $\epsilon$.

We also have

(9.13) $\quad\left|\int_{\mathbb{Q}} m_{\mathbf{r}^{-}-\mathrm{r}}(\phi f) w_{0} d x\right| \leq c\|\phi\|_{L^{\infty}}\left(\boldsymbol{r}^{\prime}-\mathrm{r}\right)$. 
Since $\phi=0$ on $S$, wo can choose $T^{\prime}$ satisfying (H5) and $\|\phi\|_{L}^{\infty}\left(T^{\prime}-T\right)$ sufficiently small. For this hole $T^{\prime}$, we have the convergence

$$
\int_{\Omega_{T_{e}^{\prime}}} \phi^{e} f^{\varepsilon} P_{\varepsilon}^{T^{\prime}} w_{\epsilon} d x \rightarrow \int_{\Omega} m_{Y-T^{\prime}}(\phi f) w_{0} d x .
$$

Combining all these results, we obtain (9.9).

Using similar arguments, we can prove the following :

Lemma (9.3). Let $\left\{w_{\epsilon}\right\}$ and $\left\{v_{\varepsilon}\right\}$ be two sequences such that

(9.15) $\left.\begin{array}{l}\left\|w_{\epsilon}\right\|_{\epsilon} \leq c \\ \left\|v_{\epsilon}\right\|_{\epsilon} \leq c\end{array}\right\}$ independent of $\epsilon$.

Then there exist a subsequence of $\epsilon$ (again denoted by $\epsilon$ ) and functions $w_{0}, v_{0}$ in $H_{0}^{1}(\Omega)$ such that we have the following convergence for this subsequence of $\epsilon$ :

$$
\left\{\begin{array}{l}
P_{\epsilon}^{T^{\prime}} w_{\epsilon} \rightarrow w_{0} \text { in } H_{0}^{1}(\Omega) \text { weak, } \\
P_{E}^{T^{\prime}} v_{\epsilon} \rightarrow v_{0} \text { in } H_{0}^{1}(\Omega) \text { weak, }
\end{array}\right.
$$

for all holes $T^{\prime}$ satisfying (H5) and

$$
\int_{\Omega_{e}} \phi^{\epsilon} f^{\varepsilon} w_{\varepsilon} v_{\epsilon} d x \rightarrow \int_{\Omega} m_{Y}(\phi f) w_{0} v_{0} d x,
$$

for all $f$ in $L^{\infty}(Y)$.

Now we have all the tools to prove the following homogenization theorem:

Theorem (9.1). We suppose that (H5) is satisfied. Let $\left(v_{\epsilon}, \mu_{\epsilon}\right)$ be a solution of the problem (5.12). We assume

$$
\text { (9.18) } \mu_{\epsilon} \rightarrow \mu \text { as } \epsilon \rightarrow 0 \text {. }
$$

Then $\mu$ is an eigenvalue of the "homogenized problem" (7.15). Furthermore there is an eigenvector $v_{0}$ in $H_{0}^{1}(\Omega)$ corres ponding to $\mu$ and a subsequence of $\epsilon$ (again denoted by $\epsilon$ ) such that

(9.19) $P_{\varepsilon}^{T^{\prime}} v_{\varepsilon} \rightarrow v_{0}$ in $H_{0}^{1}(\Omega)$ weak for all holes $T^{\prime}$

satisfying (H5), $P_{e}^{T^{\prime}}$ being the prolongation operator verifying (9.1).

\section{Proof.}

Step 1. Taking $v=v$, in (5.12), we obtain

(9.20) $\left\|v_{\varangle}\right\|_{\varangle} \leq c$, independent of $\epsilon$.

Set

$(9.21) \quad \xi_{i}=\phi^{\epsilon} \frac{\partial v_{a}}{\partial x_{i}}$ in $\Omega_{\triangleleft}, i=1,2, \ldots N$.

It follows that

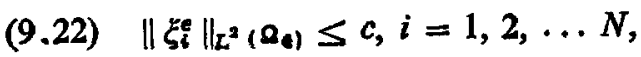

and we have

(9.23) $-\operatorname{div}\left(\phi^{\epsilon} \xi^{\epsilon}\right)=\mu_{\mathrm{s}} \phi^{\varepsilon^{2}} v_{\mathrm{g}}$ in $\Omega_{*}$ 
So, $\phi^{\epsilon} \xi^{\epsilon} . v_{\varepsilon}$ admits a trace on $\partial \Omega_{\varepsilon}$ where $v_{\varepsilon}$ is the exterior normal to $\partial \Omega_{\varepsilon}$. It is not difficult to see that

(9.24) $\phi^{\epsilon} \xi^{\epsilon}, v_{\varepsilon}=0$ on $S_{\mathrm{e}}$.

So, equation (9.23) is valid in $\Omega$ if we extend $\phi^{\epsilon}$ by zero in the holes.

(9.25) $-\operatorname{div}\left(\phi^{\epsilon} \xi^{\epsilon}\right)=\mu_{\epsilon} \phi^{\varepsilon^{2}} v_{\epsilon}$ in $\Omega$.

In (9.25), we take some arbitrary extensions for $\xi^{\epsilon}$ and $v_{\xi}$. Using Lemma (9.1), we can extract a subsequence of $\epsilon$ (again denoted by $\epsilon$ ) such that

(9.26) $\phi^{\epsilon} \xi_{i}^{e} \rightarrow \xi_{i}$ in $L^{2}(\Omega)$ weak, $i=1,2, \ldots N$.

(9.27) $P_{\epsilon}^{T^{\prime}} v_{\epsilon} \rightarrow v_{0}$ in $H_{0}^{1}(\Omega)$ weak, for all holes $T^{\prime}$ satisfying (H5).

Step 2. Using the technique of Lemma (9.2), we can pass to the limit in (9.25) to get

(9.28) $-\operatorname{div} \xi=\mu m_{Y}\left(\phi^{2}\right) v_{0}$ in $\Omega$.

Step 3. We introduce $w$ as solution to the following variational problem :

$$
\left\{\begin{array}{l}
a(\phi ; w, v)=0 \text { for } v \in V, \\
w-\Pi \in V,
\end{array}\right.
$$

where $\Pi(y)$ is a homogeneous polynomial of degree 1. Set

(9.30) $\eta=\phi \nabla w$ in $Y^{*}$.

Then $\eta$ satisfies

(9.31) $-\operatorname{div}(\phi \eta)=0$ in $Y^{*}$.

Also we have $\phi \eta \cdot v=0$ on $S$ where $v$ is the outer normal to $S$. So, we obtain (9.32) $-\operatorname{div}\left(\phi^{\epsilon} \eta^{\epsilon}\right)=0$ in $\Omega$.

We set

$$
\psi(y)=w(y)-\Pi(y) \text { for } y \in Y^{*} .
$$

Then $\psi \in V$ and we take some extension of $\psi$ in the hole and we extend $\psi$ periodically throughout $\mathbf{R}^{N}$. We define

$$
\begin{aligned}
w^{\epsilon}(x) & =\epsilon w(x / \epsilon) \\
& =\Pi(x)+\epsilon \psi(x / \epsilon) \text { in } \Omega .
\end{aligned}
$$

Since $\psi \in L^{2}(Y), \psi(x / \epsilon)$ remains bounded in $L^{2}(\Omega)$ and so (9.34) $w^{\epsilon} \rightarrow \Pi$ in $L^{2}(\Omega)$ strong.

Step 4. Let $g \in D(\Omega)$.

We take $v=g w^{\epsilon}$ in (5.12) and multiply (9.32) by $g v_{\epsilon}$ and subtract, we get

(9.35) $\int_{\Omega_{\epsilon}} \phi^{\epsilon} \xi_{i}^{\epsilon} w^{\epsilon} \frac{\partial g}{\partial x_{i}}-\int_{\Omega_{\epsilon}} \phi^{\epsilon} \eta_{i}^{\epsilon} v_{\epsilon} \frac{\partial g}{\partial x_{i}}=\mu_{\epsilon} \int_{\Omega_{e}} \phi^{\epsilon^{2}} v_{\epsilon} g w^{\epsilon} d x$. 
Using the technique of Lemma (9.2), we pass to the limit in (9.35) to get

(9.36) $\int_{\Omega} \xi_{i} \Pi \frac{\partial g}{\partial x_{i}}-\int_{\Omega} m_{Y}\left(\phi \eta_{i}\right) v_{0} \frac{\partial g}{\partial x_{i}}=\mu m_{Y}\left(\phi^{2}\right) \int_{\square} v_{0} g \Pi d x$.

It follows from $(9.36)$ and $(9.28)$ that

(9.37) $\quad \xi_{i} \frac{\partial \Pi}{\partial x_{i}}=m_{Y}\left(\phi^{2} \frac{\partial w}{\partial y_{i}}\right) \frac{\partial v_{0}}{\partial x_{i}}$ in $\Omega$.

Step 5. Now we take $\Pi=y_{j}(j=1,2, \ldots N)$. The corresponding test function $w=\psi^{(3)}+y_{\text {j }}$ where $\psi^{(3)}$ is given by $(8.9)$. So, we get

$$
\xi_{j}=m_{Y}\left(\phi^{2}\right) q_{i j} \frac{\partial v_{0}}{\partial x_{i}} \text { for } j=1,2, \ldots N
$$

This when combined with (9.28) shows that

(9.39) $A v_{0}=\mu v_{0}$ in $\Omega$.

Step 6. To complete the proof, we have to show that

$$
\text { (9.40) } \quad v_{0} \neq 0 \text {. }
$$

We have

$$
\text { (9.41) } \int_{\Omega_{e}} \phi^{\epsilon^{2}} v_{\theta}^{2}=1
$$

and at the limit we obtain

$$
m_{Y}\left(\phi^{2}\right) \int_{\Omega} v_{0}^{2}=1,
$$

and so $(9.40)$ follows.

Theorem (9.2). We assume (H5). Let $\lambda$ be the first eigenvalue of the problem (4.3) with eigenvector $\phi$. Let $\left\{v_{\varepsilon}^{l}, \mu_{\varepsilon}^{l}\right\}_{l=1}^{\infty}$ be the spectrum of the problem (5.12) Let $\left\{\lambda_{\varepsilon}^{l}\right\}_{i=1}^{\infty}$ be the sequence of eigenvalue of the problem (3.1). Then

(i) $\quad \lambda_{e}^{l}-\epsilon^{-2} \lambda=\mu_{\varepsilon}^{l}$ for $l \geq 1$.

(ii) $\quad \mu_{\epsilon}^{l} \rightarrow l$ th eigenvalue $\mu^{2}$ of the problem (7.15).

(iii) There exist a subsequence of $\epsilon$ (again denoted by $\epsilon$ ) and eigenvectors $\left\{v^{l}\right\}$ of the problem (7.15) corresponding to $\left\{\mu^{l}\right\}$ such that

(9.42) $P_{\epsilon}^{T^{\prime}} v_{\epsilon} \rightarrow v^{l}$ in $H_{0}^{1}(\Omega)$ weak for all holes $T^{\prime}$ satisfying (H5).

(9.43) $\left\{v^{i}\right\}$ form an orthogonal base in $L^{2}(\Omega)$.

(iv) If $\mu^{l}$ is a simple eigenvalue of (7.15), then given any eigenvector $v_{0}^{l}$ corresponding to $\mu^{l}$ satisfying (9.42), we can choose an eigenvector $v_{e, 0}^{l}$ of the problem (5.12) corresponding to $\mu_{\varepsilon}^{l}$ such that

(9.44) $P_{?}^{T^{\prime}} v_{\epsilon, 0}^{l} \rightarrow v_{0}^{2}$ in $H_{0}^{1}(\Omega)$ weak for the whole sequence of $\epsilon$. 
Proof. By Proposition (6.1), we can extract a subsequence of $\epsilon$ such that

(9.45) $\mu_{\varepsilon}^{l} \rightarrow \mu^{l}$ as $\epsilon \rightarrow 0$.

According to Theorem (9.1), $\mu^{l}$ is an eigenvalue of the problem (7.15) with eigenvector $v^{l}$ satisfying (9.42). To prove $\mu^{2}$ is the $l$ th eigenvalue, it suffices to verify that

(1) $\left\{v^{n}\right\}$ is an orthogonal base in $L^{2}(\Omega)$.

(2) There is no other eigenvalue except $\left\{\mu^{\eta}\right\}$ for the problem $(7.15):(u, \mu) \in$ $H_{0}^{1}(\Omega) \times \mathrm{R}$ such that $A u=\mu u$.

We remark, first, that the eigenvectors $\left\{v^{2}\right\}$ are orthogonal in $L^{2}(\Omega)$. In fact, the passage to the limit in the relation

(9.46) $\int_{\Omega_{e}} \phi^{\epsilon^{2}} v_{\epsilon}^{l} v_{\varepsilon}^{m}=\delta_{l m}$,

will give

(9.47) $m_{Y}\left(\phi^{2}\right) \int_{\Omega} v^{l} v^{m}=\delta_{l m}$.

We shall prove (2). Let $\mu$ be an eigenvalue of the problem $(7.15)$ which is different from $\mu^{l}$ with eigenvector $w$ satisfying

$$
\left\{\begin{array}{l}
A w=\mu w \text { in } \Omega, \\
\mu \neq \mu^{l} \text { for any } l, \\
\int_{\Omega} w^{2}=1 /\left(m_{Y}\left(\phi^{2}\right)\right), \\
\int_{\Omega} w v^{l}=0 \text { for each } l \geq 1 .
\end{array}\right.
$$

We can choose $l$ such that

(9.49) $\mu<\mu^{l+1}$.

Now, we define $w_{\epsilon}$ a, follows:

(9.50) $\left\{\begin{array}{l}w_{\epsilon} \in V_{\epsilon} \\ a\left(\phi^{\epsilon} ; w_{\epsilon}, v\right)=\mu\left(\phi^{\epsilon} ; w, v\right) \forall v \in V_{\epsilon} .\end{array}\right.$

The proof of Thaorem (9.1) shows that

(9.51) $\quad P_{e}^{\tau^{\prime}} w_{\epsilon} \rightarrow w$ in $H_{0}^{1}(\Omega)$ weak.

Set

(9.52) $\hat{w}_{\epsilon}=w_{\epsilon}-\sum_{i=1}^{l}\left(\phi^{\epsilon} ; w_{\epsilon}, v_{\epsilon}^{i}\right) v_{\epsilon}^{i}$.

We see easily that

(9.53) $\left(\phi^{\epsilon} ; \hat{w}_{\epsilon}, v_{e}^{i}\right)=0$ for $i=1,2, \ldots l$,

and as a consequence

(9.54) $a\left(\phi^{\epsilon} ; \hat{w}_{\epsilon}, \hat{w}_{\epsilon}\right) \geq \mu_{\epsilon}^{l+1}\left(\phi^{\epsilon} ; \hat{w}_{\epsilon}, \hat{w}_{\epsilon}\right)$. 
We can prove

$$
\left\{\begin{array}{l}
a\left(\phi^{\epsilon} ; \hat{w}_{\epsilon}, \hat{w}_{\epsilon}\right) \rightarrow \mu, \\
\left(\phi^{\epsilon} ; \hat{w}_{\epsilon}, \hat{w}_{\epsilon}\right) \rightarrow m_{Y}\left(\phi^{2}\right) \int_{\Omega} w^{2}=1 .
\end{array}\right.
$$

Hence (9.54) implies that

(9.56) $\mu \geq \mu^{i+1}$,

which is in contradiction with (9.49). This completes the proof of (2). In the same way, one can prove $v \in L^{2}(\Omega), \int_{\Omega} v v^{l}=0$ for all $l$ implies $v=0$. This proves that $v^{l}$ is the $l$ th eigenvalue of the problem (7.15).

To prove (iv). We note, first, that $\mu_{\epsilon}^{l}$ is simple for $\epsilon$ sufficiently small. In fact, since we have $\mu_{\epsilon}^{l} \rightarrow \mu^{l}$ for all $l$, the multiplicity of $\mu_{\varepsilon}^{l}$ is less than or equal to that of $\mu^{l}$. So, there are only two vectors $v_{\varepsilon, 0}^{l}$ and $-v_{e, 0}^{l}$ which satisfy (5.12). We choose one which satisfies

(9.57) $\left(\phi^{\epsilon} ; v_{\epsilon, 0}^{l}, v_{0}^{l}\right)>0$,

and this sequence must satisfy $(9.44)$.

\section{Part B: Stekloff eigenvalue problem}

\section{Problem to be treated}

With the natations introduced in $\$ 2$, we consider the following eigenvalue problem :

$$
\left\{\begin{array}{l}
\text { Find }\left(u_{\epsilon}, \lambda_{\epsilon}\right) \in W_{\epsilon} \times \mathbf{R} \text { such that } \\
-\triangle u_{\epsilon}=0 \text { in } \Omega_{\epsilon}, \\
\partial \frac{\partial u_{\epsilon}}{\partial v_{\epsilon}}=\lambda_{\epsilon} u_{\epsilon} \text { on } S_{\epsilon}, \\
u_{\epsilon} \neq 0,
\end{array}\right.
$$

where $\partial / \partial v_{\epsilon}$ is the exterior normal derivative to $S_{\epsilon}$ and

(10.2) $W_{\epsilon}=\left\{v \in H^{1}\left(\Omega_{\epsilon}\right) ; v=0\right.$ on $\left.\partial \Omega\right\}$.

Problems of the type (10.1) have been studied by Bergmann and Schiffer [4] and Bramble and Osborn [6]. This problem can be put in the variational form as follows : Consider the following problem for $g \in L^{2}\left(S_{\epsilon}\right)$ :

$$
\left\{\begin{array}{l}
a^{\epsilon}\left(w_{\epsilon}, v\right)=\int_{\boldsymbol{s}_{\epsilon}} g v \text { for } v \in W_{\epsilon}, \\
w_{\epsilon} \in W_{\epsilon} .
\end{array}\right.
$$

Set $G_{\varepsilon} g=w_{\epsilon \mid S_{\epsilon}}$. Then $G_{\epsilon}$ is self-adjoint, compact operator in $L^{2}\left(S_{\epsilon}\right)$. We can write the eigenvalue problem

$$
G_{\epsilon} u_{\epsilon}=\mu_{\epsilon} u_{\epsilon}, \mu_{\epsilon}=\frac{1}{\lambda_{\epsilon}}
$$


as follows :

$$
\left\{\begin{array}{l}
a^{\epsilon}\left(u_{\epsilon}, v\right)=\lambda_{e} \int_{S_{e}} u_{e} v \text { for } v \text { in } W_{\epsilon} \\
u_{\epsilon} \in W_{\epsilon} \\
u_{\epsilon} \neq 0
\end{array}\right.
$$

We denote by $\left\{\lambda_{\epsilon}^{l}\right\}_{l=1}^{\infty}$ the sequence of eigenvalues and by $\left\{u_{e}^{l}\right\}$ corresponding eiganveztors of this problem. We remark that $\left\{u_{e}^{l}\right\}$ form an orthogonal basis in $L^{2}\left(S_{\epsilon}\right)$. Our aim, in this part, is to study the asymptotic behaviour of $\lambda_{\varepsilon}^{2}$ and $\left\{u_{e}^{l}\right\}$ as $\epsilon \rightarrow 0$. We prove that $\left\{\epsilon^{-1} \lambda_{e}^{l}\right\} \rightarrow$ lth eigenvalue of the "homogenized problem". A very useful tool in this study will be a test function which will be defined in $\S 11$.

\section{Test function}

We define $\psi$ as the solution of the following variational problem :

(11.1) $\left\{\begin{array}{l}a(\psi, v)=-c_{0} \int_{Y^{*}} v+\int_{\mathbf{S}} v d \gamma \text { for } v \in W, \\ \psi \in W,\end{array}\right.$

where

(11.2) $W=\left\{v \in H^{1}\left(Y^{*}\right) ; v\right.$ is $Y$ periodic $\}$, and

(11.3) $\quad c_{0}=\frac{|S|}{\left|Y^{*}\right|}$

We define

(11.4) $\psi^{\epsilon}(x)=\psi(x / \epsilon)$ for $x \in \Omega_{\epsilon}$, and this will satisfy

$$
\left\{\begin{array}{l}
-\Delta \psi^{\epsilon}=c_{0} \epsilon^{-2} \text { in } \Omega_{\epsilon}, \\
\frac{\partial \psi^{\epsilon}}{\partial v_{\epsilon}}=\epsilon^{-1} \text { on } S_{\epsilon},
\end{array}\right.
$$

and so, by Green's formula we abtain

$$
\int_{\Omega_{e}} g d \gamma=\int_{\Omega_{\epsilon}} \frac{\partial \psi}{\partial y_{i}}(x / \epsilon) \frac{\partial g}{\partial x_{i}}(x)+c_{0} \epsilon^{-1} \int_{\Omega_{\epsilon}} g \text { for } g \in W_{\epsilon} .
$$

\section{Estimates on the eigenvalues}

Proposition (12.1). Let $\left\{\lambda_{\epsilon}^{l}\right\}$ be the sequence of eigenvalues of the problem (10.1). Then there exists a constant $c_{2}>0$ independent of $\epsilon$, such that

(12.1) $0<\lambda_{e}^{2} \leq c_{2} \in$ for $\epsilon>0$. 
Proof. We make use of the mini-max principle for the eigenvalues :

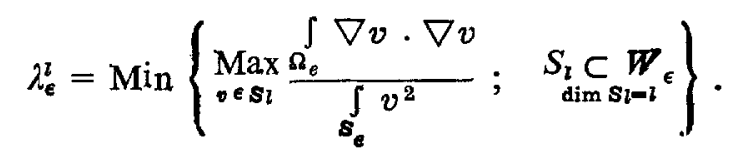

As in Proposition (6.1), we take $S_{l}$ to be the space spanned by the first $l$-eigenvectors of the problem $-\Delta w=v w$ in $\Omega,\left.w\right|_{\Omega}=0$. We get



where $v^{l}$ is the $l$ th eigenvalue of the problem (6.5).

We prove now that there exists $c_{l}$ such that

(12.4) $\operatorname{Max}_{v \in S_{l}} \frac{\int_{\mathbb{\Omega}} v^{2}}{\int_{S_{\epsilon}} v^{2}} \leq c_{l} \epsilon$.

Suppose (12.4) is not true. Then we can extract a subsequence of $\epsilon$ (again denoted by $\epsilon$ ) and $v_{\epsilon} \in S_{l}$ such that

$$
\begin{aligned}
& \text { (12.5) } \int_{\Omega} v_{\varepsilon}^{2}=1, \\
& (12.6) \in \int_{s_{e}} v_{\epsilon}^{2} \rightarrow 0 .
\end{aligned}
$$

Since $S_{l}$ is of finite dimension, the sequence $\left\{v_{\epsilon}\right\}$ remains bounded in $H_{0}^{1}(\Omega)$ also and it follows from (11.6) that

(12.7) $\int_{\mathbf{\Omega}_{6}} v_{e}^{3} \rightarrow 0$.

If $v_{\epsilon} \rightarrow v_{0}$ in $L^{2}(\Omega)$ strong, then on the one hand (12.5) implies that

$$
\int_{\Omega} v_{0}^{2}=1 \text {, }
$$

and on the other $(12.7)$ implies

$$
\int_{\mathbf{\Omega}} v_{0}^{2}=0 .
$$

This contradiction proves (12.4) and hence the Proposition.

\section{Asymptotic expansion}

Taking into account the estimates we obtained in $\$ 12$, we normalize the eigenvector of the problem $(10.1)$ as follows :

(13.1) $\int_{s_{e}} u_{e}^{2}=\epsilon^{-1}$.

Then we will have the following estimate:

(13.2) $\int_{Q_{\epsilon}} \nabla u_{\epsilon} \nabla u_{\epsilon} \leq c$, independent of $\epsilon$. 
Considering all these estimates, we propose the following Ansatz to the problem (10.1).

(13.3) $u_{\epsilon}(x)=u_{0}(x, y)+\epsilon u_{1}(x, y)+\ldots y=x / \epsilon$,

(13.4) $\lambda_{\epsilon}=\epsilon \lambda_{1}+\epsilon^{2} \lambda_{2}+\ldots$.

Each $u_{j}$ is defined on $\Omega \times Y^{*}$ and it is $Y$ perjodic in $y$. We put the expressions (13.3) (13.4) in equation (10.1). We get

(13.5) $-\left(\epsilon^{-2} \Delta_{y}+2 \epsilon^{-1} \Delta_{e y}+\Delta_{\sharp}\right)\left(u_{0}+\epsilon u_{1}+\ldots\right)=0$,

$$
\begin{aligned}
& \left(\epsilon^{-1} \frac{\partial}{\partial v_{y}}+v_{1}(y) \frac{\partial}{\partial x_{j}}\right)\left(u_{0}+\epsilon u_{1}+\ldots\right) \\
& \quad=\left(\epsilon \lambda_{1}+\epsilon^{2} \lambda_{2}+\ldots\right)\left(u_{0}+u \epsilon_{1}+\ldots\right)
\end{aligned}
$$

where $v=\left(v_{j}\right)$ is the outer normal to $S$. For the notations used in this section see $\$ 7$. Now, we equate like powers of $\epsilon$ in the above relations and we solve the resulting equations. We obtain the following results :

(13.7) $u_{0}$ is independent of $y: u_{0}(x, y)=u_{0}(x)$.

$$
u_{1}(x, y)=-\chi^{j}(y) \frac{\partial u_{0}}{\partial x}(x)+\tilde{u}_{1}(x)
$$

where $\chi^{4}$ are as defined below and $\tilde{u_{1}}$ is to be determined:

$$
\left\{\begin{array}{l}
a\left(\chi^{j}, v\right)=\int_{s} v_{j} v d \gamma \text { for } v \in W, \\
\chi^{\prime} \in W .
\end{array}\right.
$$

We get the following equation satisfied by $u_{0}$ :

$(13.10)-r_{u} \frac{\partial^{2} u_{0}}{\partial x_{i} \partial x_{i}}=\lambda_{1} u_{0}$ in $\Omega$,

with

$$
r_{i j}=\frac{1}{|S|} a\left(\chi^{4}-y_{i}, \chi^{j}-y_{i}\right) \text { for } i, j=1,2 \ldots, N .
$$

It follows from (13.11) that $\left(r_{i j}\right)$ is a symmetric, positive definite matrix and so the following eigenvalue problem (called homogenized problem in this case) is well posed :

$$
\left\{\begin{array}{l}
\text { Find }(u, \lambda) \in H_{0}^{1}(\Omega) \times \mathbf{R} \text { such that } \\
-r_{i j} \frac{\partial^{2} u}{\partial x_{i} \partial x_{j}}=\lambda u \text { in } \Omega \\
u \neq 0 .
\end{array}\right.
$$

The formal analysis so far shows that $\epsilon^{-1} \lambda_{\epsilon} \rightarrow$ to an eigenvalue of the problem (13.12). We prove this result in the later sections. 


\section{Results of homogenization}

We denote by $P_{\epsilon}$ the prolongation operator constructed from the hole $T_{\epsilon}$ and $P_{\epsilon}$ satisfies (9.1). The following Theorem is analogous to Theorem (9.1).

Theorem (14.1). Let $l \geq 1$ be an integer. Let $\lambda_{\varepsilon}^{l}$ be the $l$ th eigenvalue of the problem (10.1) with $u_{\varepsilon}^{l}$ be the corres ponding eigenvector. Then $\left\{\epsilon^{-1} \lambda_{\varepsilon}^{l}\right\}$ converges to an eigenvalue $\lambda$ of the problem (13.12) and there exists a subsequence of $\epsilon$ (denoted by $\epsilon$ ) such that

(14.1) $P_{\mathrm{a}} u_{e}^{3} \rightarrow u$ in $H_{0}^{1}(\Omega)$ weak,

where $u$ is an eigenvector of the problem (13.12) corresponding to $\lambda$.

Proof.

Step 1. We set $\lambda_{\epsilon}=\lambda_{\epsilon}^{l}, u_{\epsilon}=u_{\epsilon}^{l}$ and

(14.2) $\xi_{i}^{\epsilon}=\frac{\partial u_{\epsilon}}{\partial x_{i}}$ in $\Omega_{\epsilon}$ for $i=1,2, \ldots N$.

We put $\xi^{\epsilon}=0$ in the holes. Because of the estimates (12.1) and (13.2), we can extract a subsequence of $\epsilon$ (denoted by $\epsilon$ again) such that

(14.3) $\xi_{i}^{e} \rightarrow \xi_{i}$ in $L^{2}(\Omega)$ weak for $i=1,2, \ldots N$.

(14.4) $P_{\epsilon} u_{\epsilon} \rightarrow u$ in $H_{0}^{1}(\Omega)$ weak,

(14.5) $\epsilon^{-1} \lambda_{e} \rightarrow \lambda$.

Step 2. It follows from (10.1) that

(14.6) $\xi^{\epsilon} \cdot v_{\epsilon}=\lambda_{\epsilon} u_{\epsilon}$ on $S_{\epsilon}$,

(14.7) $\operatorname{div} \xi^{\epsilon}=0$ in $\Omega_{a}$.

We multiply equation (10.1) with $\eta \in D(\Omega)$ :

$$
\begin{aligned}
\int_{\Omega_{e}} \nabla u_{\epsilon} \nabla \eta & =\lambda_{\epsilon} \int_{\mathbf{s}_{\epsilon}} u_{\epsilon} \eta \\
& =\lambda_{\epsilon} \int_{\Omega_{e}} \frac{\partial \psi}{\partial y_{i}}(x / \epsilon) \frac{\partial}{\partial x_{i}}\left(u_{\epsilon} \eta\right)+c_{0} \epsilon^{-1} \lambda_{\epsilon} \int_{\Omega_{\epsilon}} u_{\epsilon} \eta,
\end{aligned}
$$

and the passage to the limit will give us

$$
\int_{\Omega} \xi \nabla \eta=c_{0} \lambda \int_{\Omega} \theta u \eta,
$$

which is equivalent to

(14.8) $-\operatorname{div} \xi=c_{0} \lambda \theta u$ in $\Omega$.

Step 3. We introduce $w$ as solution to the following problem :

$$
\left\{\begin{array}{l}
a(w, v)=0 \text { for } v \in W \\
w-\Pi \in W
\end{array}\right.
$$

where $\Pi(y)$ is a homogeneous polynomial of degree 1 , We put 
(14.10) $\eta=\nabla w$ in $Y^{*}$.

Then $\eta$ satisfies

(14.11) $\left\{\begin{array}{l}\operatorname{div} \eta=0 \text { in } Y^{*} \\ \eta \cdot v=0 \text { on } S\end{array}\right.$

We extend $\eta$ by zero in the hole and we put

(14.12) $\eta^{\epsilon}(x)=\eta(x / \epsilon)$ in $\Omega$.

Then we will have

(14.13) $\operatorname{div} \eta^{\epsilon}=0$ in $\Omega$.

Next we define

(14.14) $w^{\epsilon}(x)=\epsilon w(x / \epsilon)$.

As before (see Step 3, Theorem (9.1)), we get

(14.15) $w^{\epsilon} \rightarrow \Pi$ in $L^{2}(\Omega)$ strong.

Step 4. Let $g \in D(\Omega)$. Multiply (14.7) by $g w^{\epsilon}$ and (14.13) by $g P_{\epsilon} u_{\epsilon}$ and subtract :

(14.16) $-\int_{\Omega_{\epsilon}} \xi_{i}^{e} \frac{\partial g}{\partial x_{i}} w^{\epsilon}+\int_{\Omega_{e}} \eta_{i}^{e} \frac{\partial g}{\partial x_{i}} P_{\epsilon} u_{\epsilon}+\int_{\mathbf{s}_{\epsilon}} \lambda_{\epsilon} u_{\epsilon} g w^{\epsilon}=0$.

We can pass to the limit directly in the first two terms. For the third we use the formula (11.6). We get

$(14.17)-\int_{\boldsymbol{\Omega}} \xi_{i} \frac{\partial g}{\partial x_{i}} \Pi+\int_{\boldsymbol{\Omega}} m_{Y}\left(\chi \frac{\partial w}{\partial y_{i}}\right) \frac{\partial g}{\partial x_{i}} u+c_{0} \lambda \theta \int_{\boldsymbol{\Omega}} u g \Pi=0$

which gives

(14.18) $\quad \xi_{i} \frac{\partial \Pi}{\partial x_{i}}=m_{Y}\left(\chi \frac{\partial w}{\partial y_{i}}\right) \frac{\partial u}{\partial x_{i}}$.

Step 5. Now we take $\Pi=y_{1}(j=1,2 \ldots N)$, The corresponding test function $w=-\chi^{(j)}+y$, where $\chi^{(j)}$ is defined by (13.9). So we get

(14.19) $\xi_{i}=\frac{1}{|Y|} \int_{Y^{*}}\left(\delta_{i j}-\frac{\partial \chi^{(f)}}{\partial y_{i}}\right) \frac{\partial u}{\partial x_{i}}$,

$$
=\frac{|S|}{|Y|} r_{i j} \frac{\partial u}{\partial x_{i}}
$$

Combining this with (14.8) we obtain

(14.20) $-r_{i j} \frac{\partial^{2} u}{\partial x_{i} \partial x_{j}}=\lambda u$. 
Step 6. To complete the proof, we have to show that $u \neq 0$. To see this, we take $g=u_{e}^{2}$ in (11.6) :

$$
\epsilon \int_{\mathbf{S}_{\epsilon}} u_{\epsilon}^{2}=c_{0} \int_{\mathbf{\Omega}_{\boldsymbol{\epsilon}}} u_{e}^{2}+\epsilon \int_{\mathbf{\Omega}_{\boldsymbol{E}}} \frac{\partial \psi}{\partial y_{i}}(x / \epsilon) \frac{\partial}{\partial x_{i}}\left(u_{\varepsilon}^{2}\right) .
$$

Using (13.1), wa pass to the limit in this relation and we obtain

(14.21) $1=c_{0} \theta \int_{\Omega} u^{2}$,

from which it fallows that $u \neq 0$.

We now give our final result in the Stekloff case.

Theorem (14.2)

Let $\left\{\lambda_{e}^{l}\right\},\left\{u_{\epsilon}^{l}\right\}$ be the sequences of eigenvalues and eigenvectors of the problem (10.1). We suppose that the eigenvectors are normalized according to (13.1): $\int_{S_{e}} u_{e}^{2}=\epsilon^{-1}$. Then

(i) $\lambda_{\varepsilon}^{l} \rightarrow \lambda^{l}$, the $l$ th eigenvalue of the problem (13.12).

(ii) there exists a subsequence of $\epsilon$ (again denoted by $\epsilon$ ) such that

(14.22) $P_{\varepsilon} u_{e}^{x} \rightarrow u^{2}$ in $H_{0}^{1}(\Omega)$ weak,

where $u^{l}$ satisfies $(13.12)$ :

$$
-r_{i} \frac{\partial^{2} u}{\partial x_{i} \partial x_{j}}=\lambda^{2} u \text { in } \Omega,\left.u\right|_{\partial \Omega}=0 .
$$

(iii) If $\lambda^{l}$ is a simple eigenvalue of the problem (13.12) then given any eigenvector $v^{i}$ associated to $\lambda^{i}$ satisfying (14.2), we can choose an eigenvector $v_{e}^{l}$ of the problem (10.1) corresponding to $\lambda_{e}^{L}$ such that

(14.23) $\int_{s_{e}} v_{e}^{l^{2}}=\epsilon^{-1}$

(14.24) $P_{\epsilon} v_{e}^{l} \rightarrow v^{I}$ in $H_{0}^{1}(\Omega)$ weak for the whole sequence.

Proof. From the previous Theorem, we know that (ii) is true for a subsequence of $\epsilon$. We obtain also the orthogonality condition for the eigenvectors by means of the method of Step 6 of Theorem (14.1):

$$
\int_{\Omega} u^{l} u^{m}=\frac{1}{c_{0} \theta} \delta_{l_{m}} \text { for } l, m \geq 1 \text {. }
$$

Finally, it remains to prove that the limiting point $\lambda^{l}$ of $\left\{\lambda_{e}^{l}\right\}$ is the $l$ th eigenvalue of (13.12). For this, it suffices to verify that

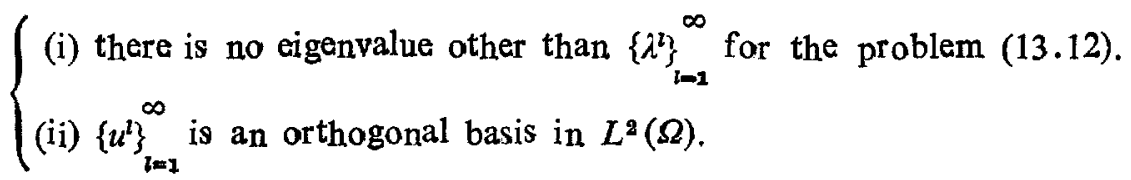


In the following, we verify (14.26) (i) The proof of (14.26) (ii) is analogous.

Let us suppose that there is an eigenvalue $\mu$ different from $\lambda^{l}$ with eigenvector $u$ :

(14.27) $\left\{\begin{array}{l}-r_{u j} \frac{\partial^{2} u}{\partial x_{i} \partial x_{j}}=\mu u \text { in } \Omega, \\ u \in H_{\mathrm{o}}^{1}(\Omega), \\ \mu \neq \lambda^{2} \text { for all } l, \\ \int_{\Omega} u u^{l}=0 \text { for each } l, \\ \int_{\Omega} u^{2}=1 .\end{array}\right.$

We can choose an integer $l$ such that

(14.28) $\mu<\lambda^{4+1}$.

We define $w_{\epsilon}$ as the solution of

$$
\left\{\begin{array}{c}
-\Delta w_{\epsilon}=0 \text { in } \Omega_{\epsilon} \\
w_{\epsilon} \in W_{\epsilon} \\
\frac{\partial w_{\epsilon}}{\partial v_{\epsilon}}=\epsilon \mu u \text { on } S_{\epsilon}
\end{array}\right.
$$

From the proof of Theorem (14.1), it is seen that

(14.30) $P_{\epsilon} w_{\epsilon} \rightarrow u$ in $H_{0}^{1}(\Omega)$ weak.

Now consider the element

(14.31) $v_{\epsilon}=w_{\epsilon}-\epsilon \sum_{k=1}^{l}\left[\int_{S_{e}} w_{\epsilon} u_{e}^{k}\right] u_{e}^{k}$

Since

$$
\int_{s_{\varepsilon}} v_{\epsilon} u_{\epsilon}^{k}=0 \text { for } k=1,2, \ldots, l
$$

we have

(14.32) $a^{\epsilon}\left(v_{\epsilon}, v_{\epsilon}\right) \geq \lambda_{e}^{l+1} \int_{s_{e}} v_{e}^{2}$.

We now pass to the limit in (14.32) using (11.6). We have, first

$$
\epsilon \int_{s_{e}} w_{\epsilon} u_{\epsilon}^{k}=\epsilon \int_{\Omega_{e}} \frac{\partial \psi}{\partial y_{i}}(x / \epsilon) \frac{\partial}{\partial x_{i}}\left(w_{\epsilon} u_{\varepsilon}^{k}\right)+c_{0} \int_{\Omega_{e}} w_{\epsilon} u_{e}^{k},
$$

and so

$$
\epsilon \int_{S_{\varepsilon}} w_{\epsilon} u_{\epsilon}^{k} \rightarrow c_{0} \int_{\Omega} \theta u u^{k}=0
$$

As a consequence,

(14.33) $P_{\epsilon} v_{E} \rightarrow u$ in $H_{0}^{1}(\Omega)$ weak. 
Using this and taking $g=\lambda_{e}^{2+1} v_{\epsilon}^{2}$ in (11.6), we get

(14.34) $\lambda_{e}^{2+1} \int_{\mathbf{s}_{\epsilon}} v_{e}^{2} \rightarrow c_{0} \lambda^{l+1} \theta$.

By similar arguments, we prove that

(14.35) $a^{\epsilon}\left(v_{\epsilon}, v_{\epsilon}\right) \rightarrow c_{0} \theta \mu$.

Now passing to the limit in (14.32) we get

(14.36) $\mu \geq \lambda^{3+1}$

which contradicts $(14.28)$.

To prove (iii), we remark that $\lambda_{e}^{l}$ is simple for sufficiently small $\epsilon$ if $\lambda^{l}$ is so. Afterwards, it suffices to pick up an eigenvector $v_{\varepsilon}^{l}$ associated to $\lambda_{\varepsilon}^{l}$ such that

(14.37) $\int_{s_{e}} v_{e}^{l} v^{b}>0$.

\section{Part C: Neumann eigenvalue problem}

\section{Problem to be studied}

We consider the following eigenvalue problem

$$
\left\{\begin{array}{l}
\text { Find }\left(u_{\epsilon}, \lambda_{\epsilon}\right) \in W_{\epsilon} \times \mathbf{R} \text { such that } \\
-\Delta u_{\epsilon}=\lambda_{\epsilon} u_{\epsilon} \text { in } \Omega_{\epsilon}, \\
\partial u_{\epsilon}=0 \text { on } S_{\epsilon}, \\
\partial v_{\epsilon} \\
\int_{\epsilon} u_{\epsilon}^{2}=1,
\end{array}\right.
$$

where we have used the notations of $\S 2$ and $\S 10$. Let us denote by $\left\{\lambda_{e}^{l}\right\}$ and $\left\{u_{e}^{l}\right\}$ the sequences of eigenvalues and the corresponding eigenvectors of the problem (15.1). We know that

$$
\text { (15.2) }\left\{\begin{array}{l}
\lambda_{\epsilon}^{1}<\lambda_{e}^{2} \leq \lambda_{e}^{3} \ldots \rightarrow \infty, \\
\left\{u_{\epsilon}^{l}\right\} \text { form an orthonormal basis in } L^{2}\left(\Omega_{\epsilon}\right) \text {. }
\end{array}\right.
$$

Here, we study the asymptotic behaviour of $\lambda_{e}^{l}$ and $u_{e}^{l}$ as $\epsilon \rightarrow 0$. We prove that $\left\{\lambda_{e}^{l}\right\}$ converges to the 7 th eigenvalue of the "homogenized problem".

Since the method followed here is similar to Stekloff case, we do not give details of the proofs.

\section{Estimates on the eigenvalues}

Proposition (16.1). Let $\lambda_{e}^{l}$ be the $/$ th eigenvalue of the problem (15.1). Then there exists a constant $c_{z}$ independent of $\epsilon$ such that

$$
\lambda_{e}^{l} \leq c_{i}
$$


Proof. As in the proof of Proposition (12.1), by the use of mini-max principle we get

(16.1) $\lambda_{e}^{l} \leq v^{l} \operatorname{Max}_{\bullet \in s_{l}} \frac{\int_{\Omega} v^{2}}{\int_{\Omega_{e}} v^{2}}$.

But we saw in the course of the proof of Proposition (12.1) that the right side of (16.1) is bounded above independently of $\epsilon$ and this completes the proof.

\section{Asymptotic analysis}

With the help of the estimates in $\$ 16$ and the normalization condition for the eigenvectors, we propose the following Ansatz for the problem (15.1) :

$$
u_{\epsilon}(x)=u_{0}(x, y)+\epsilon u_{1}(x, y)+\ldots, y=x / \epsilon,
$$

(17.2) $\lambda_{\varepsilon}=\lambda_{0}+\epsilon \lambda_{1}+\ldots$

where $u_{j}$ is defined on $\Omega \times Y^{*}$ and it is $Y$ periodic in $y$.

We substitute these expressions in equation ${ }^{*}(15.1)$ :

$$
\begin{gathered}
-\left(\epsilon^{-2} \triangle_{y}+2 \epsilon^{-1} \triangle_{x y}+\triangle_{*}\right)\left(u_{0}+\epsilon u_{1}+\ldots\right) \\
=\left(\lambda_{0}+\epsilon \lambda_{1}+\ldots\right)\left(u_{0}+\epsilon u_{1}+\ldots\right),
\end{gathered}
$$

$$
\left(\epsilon^{-1} \frac{\partial}{\partial v_{y}}+v_{j} \frac{\partial}{\partial x_{i}}\right)\left(u_{0}+\epsilon u_{i}+\ldots\right)=0 .
$$

As before, we equate the powers of $\epsilon$ on either side and we obtain the following results :

(17.5) $u_{0}$ is independent of $y: u_{0}(x, y)=u_{0}(x)$.

$$
u_{1}(x, y)=-\chi^{j}(y) \frac{\partial u_{0}}{\partial x_{j}}(x)+\tilde{u}_{1}(x)
$$

where $\chi^{f}$ are defined in (13.9), We get the following equation satisfied by $u_{0}$ and $\lambda_{0}$ :

$$
-s_{i j} \frac{\partial^{2} u_{0}}{\partial x_{i} \partial x_{j}}=\lambda_{0} u_{0} \text { in } \Omega
$$

with

$$
s_{i j}=\frac{1}{\left|Y^{*}\right|} a\left(\chi^{i}-y_{i}, \chi^{j}-y_{j}\right) \text { for all } i, j
$$

So, the corresponding "homogenized problem" in this caso can be formulated as follows :

$$
\left\{\begin{array}{c}
\text { Find }(u, \lambda) \in H_{0}^{1}(\Omega) \times \mathbf{R} \text { such that } \\
-s_{i j} \frac{\partial^{2} u}{\partial x_{i} \partial x_{j}}=\lambda u \text { in } \Omega \\
u \neq 0 .
\end{array}\right.
$$


In the following section, we justify the formal calculations done above.

\section{Homogenization theorem}

The proof of the following result proceeds along the lines of Thearems (14.1) and (14.2) and therefore omitted.

Theorem (18.1). Let $\left\{\lambda_{e}^{l}\right\},\left\{u_{e}^{l}\right\}$ be the sequences of eigenvalues and the corresponding eigenvectors of the problem (15.1) satisfying (15.2). Then

(i) $\lambda_{\varepsilon}^{2} \rightarrow \lambda^{i}$, the $l$ th eigenvalue of the problem (17.9).

(ii) there exists a subsequence of $\epsilon$ (denoted again by $\epsilon$ ) such that

$P_{e} u_{e}^{\tau} \rightarrow u^{l}$ in $H_{0}^{1}(\Omega)$ weak,

where $u^{l}$ is an eigenvector corresponding to $\lambda^{l}$.

(iii) If $\lambda^{l}$ is simple, then given any eigenvector $v^{l}$ corresponding to $\lambda^{l}$ such that (18.1) $\int_{\Omega} v^{\imath^{2}}=1 / \theta$,

we can choose an eigenvector $v_{e}^{l}$ of the problem (15.1) corresponding to $\lambda_{e}^{l}$ such that $P_{\mathrm{c}} v_{e}^{\imath} \rightarrow v^{l}$ in $H_{0}^{1}(\Omega)$ weak for the whole sequence of $\epsilon$.

\section{Correctors and error estimates}

In this paragraph, we briefly mention a method of finding correctors of first order for simple eigenvalue of the Neumann problem. We will also see that the problem of error estimates is reduced to that of stationary problem for which we refer to Lions [13]. For the study of correctors in the homogenization theory, we refer to Bensoussan et al [3], Bourgat and Dervieux [5], Kesavan [12].

We consider a simple eigenvalue $\lambda_{0}$ of the problem (17.9) with eigenvector $u_{0}$ satisfying

(19.1) $\int_{\Omega} u_{0}^{2}=1 / \theta$.

Let $u_{\epsilon}, \lambda_{\epsilon}$ be eigenvector and eigenvalue of the problem (15.1) satisfying

(19.2) $P_{\epsilon} u_{\epsilon} \rightarrow u_{0}$ in $H_{0}^{1}(\Omega)$ weak for the whole sequence of $\epsilon$,

(19.3) $\lambda_{c} \rightarrow \lambda_{0}$.

Let us define $w_{\epsilon}$ as the solution of

(19.4) $\left\{\begin{array}{l}-\Delta w_{\epsilon}=\lambda_{0} u_{0} \text { in } \Omega_{\epsilon}, \\ w_{\epsilon} \epsilon W_{\epsilon}, \\ \frac{\partial w_{\epsilon}}{\partial v_{\epsilon}}=0 \text { on } S_{\epsilon} .\end{array}\right.$

The proof of Theorem (18.1) shows that

(19.5) $P_{e} w_{\epsilon} \rightarrow u_{0}$ in $H_{0}^{1}(\Omega)$ weak. 
Multiplying (19.4) by $u_{\epsilon}$ and (15.1) by $w_{\epsilon}$, we obtain

(19.6) $\lambda_{\epsilon}\left(u_{\star}, w_{\epsilon}\right)_{\Omega_{\epsilon}}=\lambda_{0}\left(u_{0}, u_{\epsilon}\right)_{\Omega_{\epsilon}}$,

where $(., .)_{\Omega_{\varepsilon}}$ denotes the scalar product in $L^{2}\left(\Omega_{\epsilon}\right)$.

First let us prove the following:

Theorem (19.1)

With $\lambda_{0}, u_{0}, \lambda_{\epsilon}, u_{\epsilon}, w_{\epsilon}$ defined as above, we have

(19.7) $\left|\lambda_{\epsilon}-\lambda_{0}\right| \leq c\left\|w_{\epsilon}-u_{0}\right\|_{L^{2}\left(\Omega_{\epsilon}\right)}$,

for sufficiently small $\epsilon$ and where $c>0$ is a constant independent of $\epsilon$.

Proof. We have

$$
\lambda_{\epsilon}-\lambda_{0}=\frac{\lambda_{0}}{\left(u_{\epsilon}, w_{\epsilon}\right)_{\Omega_{\epsilon}}}\left(u_{\epsilon}, u_{0}-w_{\epsilon}\right)_{\Omega_{\epsilon}} .
$$

But $\left(u_{\epsilon}, w_{\epsilon}\right)_{\Omega_{\epsilon}} \rightarrow 1$ as $\epsilon \rightarrow 0$ and so we obtain (19.7).

Now, we give a result which estimates the error between the eigenvectors by choosing an eigenvector corres ponding to $\lambda_{\epsilon}$. For that, we first define $z_{\epsilon}$ uniquely as follows :

$$
\left\{\begin{array}{l}
a^{e}\left(z_{\epsilon}, v\right)-\lambda_{\epsilon}\left(z_{\epsilon}, v\right)_{\Omega_{\epsilon}}=\lambda_{\varepsilon}\left(u_{\varepsilon}, v\right)_{\Omega_{\varepsilon}}-\lambda_{0}\left(u_{0}, v\right)_{\Omega_{\epsilon}} \text { for } v \in W_{\epsilon} \\
z_{\epsilon} \in W_{\epsilon} \\
\left(z_{\varepsilon}, u_{\varepsilon}\right)_{\Omega_{\varepsilon}}=0
\end{array}\right.
$$

Then

(19.9) $\tilde{u}_{*}=z_{\text {s }}+w_{\text {s }}$

$i_{s}$ an eigenvector of the problem (15.1) corresponding to $\lambda_{\varepsilon}$. We have the following estimate for $z_{\mathbf{e}}$ :

Lemma (19.1). There is a constant $c>0$ independent of $\epsilon$ such that

(19.10) $\left|z_{\epsilon}\right|_{1}, \Omega_{\epsilon} \leq c\left\|\lambda_{e} w_{e}-\lambda_{0} u_{0}\right\|_{L^{2}\left(\Omega_{e}\right)}$.

Proof. Suppose $(19.10)$ is not true. Then there will be a subsequence of $\epsilon$ (again denoted by $\epsilon$ ) such that

(19.11) $\left|z_{\epsilon}\right| 1, \Omega_{\epsilon}=1$,

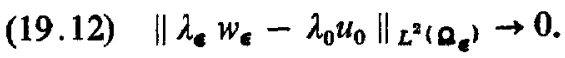

We can pass to the limit in (19.8) as we did in equation (15.1). We obtain (19.13) $P_{a} z_{\star} \rightarrow z_{0}$ in $H_{0}^{1}(\Omega)$ weak,

where $z_{0}$ satisfies

$$
\left\{\begin{array}{c}
-s_{i j} \frac{\partial^{2} z_{0}}{\partial x_{i} \partial x_{j}}=\lambda_{0} z_{0} \text { in } \Omega \\
\int_{\mathbb{Q}} z_{0} u_{0}=0
\end{array}\right.
$$


Since $\lambda_{0}$ is simple, this implies that

(19.15) $z_{0}=0$.

On the other hand, taking $v=z_{\epsilon}$ in (19.18), we get

$$
1=\lambda_{\epsilon}\left(z_{\epsilon}, z_{\epsilon}\right)_{\Omega_{\varepsilon}}+\left(\lambda_{\epsilon} w_{\epsilon}-\lambda_{0} u_{0}, z_{\epsilon}\right)_{\Omega_{\epsilon}},
$$

which, at the limit, gives a contradiction.

Theorem (19.2)

Let $\lambda_{0}, \lambda_{\epsilon}, w_{\epsilon}, u_{0}, \tilde{u}_{\epsilon}$ be defined as above. Then there is a constant $c>0$ independent of $\epsilon$ such that

(19.16) $\left\|\tilde{u}_{e}-u_{0}\right\|_{L^{2}\left(\Omega_{\epsilon}\right)} \leq c\left\|w_{\epsilon}-u_{0}\right\|_{L^{2}\left(\Omega_{\epsilon}\right)}$.

Proof. We write

$$
\tilde{\epsilon}_{\epsilon}-u_{0}=z_{\epsilon}+w_{\epsilon}-u_{0} \text {. }
$$

The proof is completed by using (19.11) and Theorem (19.1) and the fact that

$$
\left\|z_{\epsilon}\right\|_{L^{2}\left(\Omega_{\epsilon}\right)} \leq \mathcal{C}\left|z_{\epsilon}\right|_{1},\left(\Omega_{\epsilon}\right) .
$$

Now we give a first order corrector for the eigenvalue $\lambda_{\varepsilon}$. We use (19.6). We express

$$
\begin{aligned}
& \lambda_{\epsilon}=\lambda_{0}+\epsilon \lambda_{1}+\ldots \\
& u_{\epsilon}=u_{0}+\epsilon u_{1}+\ldots \\
& w_{\epsilon}=u_{0}+\epsilon w_{1}+\ldots
\end{aligned}
$$

We put these expressions in (19.6) and identify powers of $\epsilon$ : we get

$$
\lambda_{1}=-\lambda_{0} \frac{\left(u_{0}, w_{1}\right) \Omega_{\epsilon}}{\left(u_{0}, u_{0}\right) \Omega_{\epsilon}}
$$

where $w_{1}$ is the first order corrector for $w_{\epsilon}$. The estimate we give below is better than that given by Theorem (19.1).

\section{Theorem (19.3)}

Let $\lambda_{\epsilon}, \lambda_{0}, w_{\varepsilon}, u_{0}$ be as in the previous Theorem. Let $\lambda_{1}$ be [defined by (19.17). Then we have

$$
\begin{aligned}
& \left|\lambda_{\epsilon}-\lambda_{0}-\epsilon \lambda_{1}\right| \leq c\left\{\epsilon\left\|w_{\epsilon}-u_{0}\right\|_{L^{2}\left(\Omega_{\epsilon}\right)}+\left\|w_{\epsilon}-u_{0}\right\|^{2} L^{2}\left(\Omega_{\epsilon}\right)\right. \\
& \left.+\left\|w_{\epsilon}-u_{0}-\epsilon w_{1}\right\|_{L^{2}\left(\Omega_{\epsilon}\right)}\right\},
\end{aligned}
$$

where $c$ is a constant independent of $\epsilon$. 
Proof. We have

$$
\begin{aligned}
\lambda_{\epsilon}- & \lambda_{0}-\epsilon \lambda_{1}=\frac{\lambda_{0}}{\left(u_{0}, u_{0}\right)_{\Omega_{\epsilon}}}\left(u_{0}, u_{0}+\epsilon w_{1}-w_{\epsilon}\right)_{\Omega_{\epsilon}} \\
& -\left(\lambda_{\epsilon}-\lambda_{0}\right) \frac{\left(u_{0}, \epsilon w_{1}\right) \Omega_{\epsilon}}{\left(u_{0}, u_{0}\right) \Omega_{\epsilon}}+\left[\frac{\lambda_{\epsilon}}{\left(u_{0}, u_{0}\right) \Omega_{\epsilon}}\left(u_{0}, w_{\epsilon}\right)_{\Omega_{\epsilon}}-\lambda_{0}\right] .
\end{aligned}
$$

We only have to estimate the last term. But using (19.6), one can express the last term as follows:

$$
\begin{gathered}
\frac{\lambda_{\epsilon}}{\left(u_{0}, u_{0}\right) \Omega_{\epsilon}}\left(u_{0}, w_{\epsilon}\right) \Omega_{\epsilon}-\lambda_{0}=\frac{\lambda_{\epsilon}}{\left(u_{0}, u_{0}\right) \Omega_{\epsilon}}\left(u_{0}-\tilde{u}_{\epsilon}, w_{\epsilon}-u_{0}\right) \Omega_{\epsilon} \\
+\frac{\lambda_{\epsilon}-\lambda_{0}}{\left(u_{0}, u_{0}\right) \Omega_{\epsilon}}\left(u_{0}, u_{0}-\tilde{u}_{\epsilon}\right) \leq c\left\|w_{\epsilon}-u_{0}\right\|^{2} L^{2}\left(\Omega_{\epsilon}\right),
\end{gathered}
$$

by Theorems (19.1) and (19.2). This completes the proof.

\section{Acknowledgements}

The author is thankful to Professor J L Lions, College de France, Paris, for his help and encouragement in carrying out this work during his stay at Paris. The discussions, the author had with Professor L Tartar, Orsay, France, were very fruitful.

The author thanks the referee for having brought Rauch's article "The Mathematical Theory of Crushed Ice", Partial Differential Equations and Related topics, Springer Verlag, Lecture Notes in Mathematics, No. 446, 1975, to his notice. In this article, Rauch considers what he calls the crushed ice problem; obviously this is related to our Dirichlet problem. Rauch shows, in dimension 3, that the first eigenvalue $\lambda_{1} \rightarrow \infty$ when $n \rightarrow \infty, r \rightarrow 0, n r^{3}=$ constant and $n r^{2} \rightarrow \infty$ where $n$ is the number of holes and $r$ is the size of each hole. In our case, $n=0\left(\epsilon^{-3}\right), r=0(\epsilon)$ and so $n r^{2}=0\left(\epsilon^{-1}\right) \rightarrow \infty$ as $\epsilon \rightarrow 0$. Therefore, it is not surprising that $\lambda_{1} \rightarrow \infty$ for the Dirichlet problem. However, our method even gives an asymptotic expansion valid not only for the first eigenvalue but also for all eigenvalues.

\section{References}

[1] Baouendi M S 1967 Bull. Soc. Math. de France 9545

[2] Baouendi M S and Goulaouic C 1968 C.R. Acad. Sci. Seric A tome 266336

[3] Bensoussan A, Lions J L and Papanicolaou G 1978 Asymptotic methods in periodic structures (Amsterdam: North Holland)

[4] Bergmann S and Schiffer M 1953 Kernel functions and elliptic differential equations in mathematical physics (New York: Academic Press)

[5] Bourgat J F and Dervieux A 1978 Etude des correcteurs provenant du development asymptotique, Rapport Laboria No. 278 
[6] Bramble J H and Osborn J E 1972 in The mathematical foundations of the finite element method with applications to the partial differential equations (ed. : A K Aziz) (New York : Academic Press)

[7] Cioranescu D 1978 Sur quelques equations aux derivees partielles posees par la Mechanique des milieux continus, These d'Etat Paris.

[8] Cioranescu D and Saint Jean Paulin J 1978 Homogeneisation dans des ouverts avec cavite's reparties periodiquement, Rapport de l'universite $P$ et $M$ Curie, Paris

[9] Duvaut G 1977 Comportement macroscopique d'une plaque perforee periodiquement, Lecture Notes (Berlin : Springer-Verlag) 594

[10] Geymonat G and Grisvard P 1967 Le Matematiche 222

[11] Goudjo C 1970 Problems aux limites dans les espaces de Sobolov avec poids These de 3 eme cycle Nice

[12] Kesavan S 1979 Approximation des problemes de valens propres lineaires et non-lineaires These d'Etat, Paris

[13] Lions J L 1975-77 Cours d'homogeneisation, College de France

[14] Tartar L 1977 Problems d'homogeneisation dans les equations aux derivees particlles, College de France Cours Peccot

[15] Vanninathan M 1978 C.R. Acad. Sci. Paris Serie A tome 287 p. 823

[16] Vanninathan M 1978 C.R. Acad. Sci. Serie A tome 287 p. 403

[17] Weinstein A and Steinger W 1972 Methods of intermediate problems for eigenvalues. Theory and ramifications (New York: Academic Press) 